\title{
Drilling in the Water by "Creating a Safe and Free Passage for the Movement of People and Equipment between the Surface and the Bottom", Using Several Methods for Extracting Natural Resources and Scientific Research
}

\author{
Walid Nabil (1) \\ Department of Geography, Cairo University, Cairo, Egypt \\ Email: waleednabilalin@yahoo.com
}

How to cite this paper: Nabil, W. (2021) Drilling in the Water by "Creating a Safe and Free Passage for the Movement of People and Equipment between the Surface and the Bottom", Using Several Methods for Extracting Natural Resources and Scientific Research. Journal of Geographic Information System, 13, 210-242.

https://doi.org/10.4236/jgis.2021.132012

Received: March 2, 2021

Accepted: April 17, 2021

Published: April 20, 2021

Copyright $\odot 2021$ by author(s) and Scientific Research Publishing Inc. This work is licensed under the Creative Commons Attribution International License (CC BY 4.0).

http://creativecommons.org/licenses/by/4.0/

\begin{abstract}
The research develops a new plan that follows a precise system for drilling at the bottom of the water, by creating a "digging corridor" that allows people to move from the top of the surface of rivers or seas and oceans to the bottom, allowing all the huge mechanical equipment to be lowered to continue drilling at the bottom vertically, until reaching rocky soil; in order to change the drilling paths and "develop the attack", and establish long corridors to obtain the dysfunctional economic wealth, the project can be used in scientific research and the establishment of cities in the future below the seabed. There are different characteristics of drilling in fresh and salt water, due to the difference in water quality, current speed and nature soil analysis, erosion power, and erosion. The research was divided between easy ways to reach simple depths (10 - 20 meters), using various cheap means, and strategies for establishing "drilling corridors" at medium (more than 20 - less than 100 meters) and large depths (from 100 meters - less than 1 kilometer), which depends on the construction of a concrete building with a large base, which enables it to successfully perform its role, and at great depths (more than 1 kilometer) require adding more supports to the building, and emergency plans have been taken care of. It is important to establish strong electronic doors to protect tunnel passages, such as: closing them at the time of danger, preparing sectors for evacuation, and the possibility of selling the "digging corridor" and tunnels, or gifting them to scientists, or moving them and closing or blowing up the underpasses. In conclusion, this research will contribute to "opening a new environment", to the activity of Wan Humans divorce in the future.
\end{abstract}




\section{Keywords}

Construction, Path, Excavation, Design, Engineering, New Road, Opening, Path

\section{Background}

There was a dream one day that: "Will a person be able to perform a water splitting operation? Cut and separate it from each other, and prevent its parts interconnected?", and many gains after safe access to the bottom, and the continuation of drilling to achieve great success in the exploration of natural resources, and awe may encounter some prehistoric relics, the digging tunnels may be used to construct tunnels and roads below the sea, and to contribute to the establishment of cities below the water.

This scientific paper is one of the possible attempts to stop the bonding of water and neutralize its role to reach the bottom, and we still have a lot of convictions that man will make many successful attempts in this regard using other technological methods, including centrifugation, and magnetic repulsion, and the use of electricity to achieve a repulsion between water molecules, to cause the water to diverge from each other, appearing like gaps in the water, and perhaps science can expand it to the maximum depth until it reaches the bottom.

A number of means and tools for safe access to the bottom will be provided here, using the simplest, cheapest and best types of technology, and despite this there will be a lot of expenses for the success of the project, but it is hoped that the results of the project and exploration operations will achieve the bottom in the payment of expenses and achieving profits in a way that encourages to do more of it.

What are the most appropriate methods used in the beginning to reach the bottom of rivers and seas? Who to choose?

The environment alone is the one that chooses and imposes the means designed to suit it. In this case, the most decisive thing is the "water depth range". If the water depth is less than about 10 meters, then it is sufficient to use "several inverted balloons", and if the water depth is between $10-100$ meters, it is better to use "concrete rings". If the water depth exceeds 1 kilometer or more, it is better to use several common means consisting of long iron pipes + concrete rings. It may also be necessary to provide a "submarine equipped for drilling".

These points will be studied.

* First. The expected huge advantages of underwater drilling.

* Second: Characteristics of drilling in fresh and salt water.

* Third: The strategy of safe access to a small water depth, especially (10 20 meters) in rivers, through several balloons.

* Fourth: The strategy of drilling in medium-depth water (from 20 to 100 meters).

* Fifth: The strategy of drilling in medium-depth waters (more than 100 
meters).

* Sixth: The strategy of drilling in a marine environment to reach a depth of $1 \mathrm{~km}$ or more.

* Seventh: Safety and emergency strategy.

* Eighth: Procedures for the complete and final disposal of drilling mines in water.

* Conclusion.

* References.

\section{First: The Expected Huge Advantages of Underwater Drilling}

We were not able to enumerate many of the advantages, gains and wealth that would be achieved if a person succeeded in securing access to the water floor, and with him all his tools and mechanical means and others, but it was possible to list the most prominent possible points, which are:

Features of drilling in the bottom of the river currents.

1) Construction of tunnels for the passage of cars and pedestrians.

If successful drilling is done at the bottom of the river and it is possible to reach a large depth of more than 30 - 40 meters of drilling under the river, so that there is no leakage or falling of water particles in the tunnels, it is possible to start digging towards the sides of the river The right bank and the left bank, and the continuation of digging to reach a point farther from the river by more than approximately 200 meters at the very least (to be reassured in moving away from riverine influences and flood paths and their potential range of movement) to gradually dig

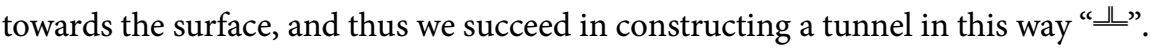

And if we want to create a tunnel that connects the two river banks and allows cars and pedestrians to cross the river from the tunnel safely; We recommend pumping a layer of strong concrete at the bottom of the tunnel, with an appropriate thickness (at least $30-40 \mathrm{~cm}$ to bear the weight of the possibility of collapsing parts of the roof) and consider this concrete layer as the "tunnel roof", where a gap is prepared in the concrete for drilling beneath it regularly, so that it does not increase The diameter of the tunnel is more than the concrete roof that protects it, and does not cause the concrete to collapse over it, and because what is required to reach a large diameter of the tunnel, which will cause the collapse of the roof, therefore every part of the tunnel is dug and then closed after the completion of the concrete pouring, and of course safety precautions are taken in drilling and weld the concrete together to turn For one block that works with strength, regularity and potency.

2) Trace the paths of the gold granules.

One advantage of drilling down fresh water is that it follows the path of grains of gold in the course of rivers. And the extraction of layers bearing the most economic concentrations of the gold metal, which have accumulated under the modern river sediments, so if we know that rivers are in their youth stage where the water flow increases and the subsequent high rate of river sculpture at the 
bottom turns the riverbed into a letter " $\mathrm{V}$ ", and that with the river reaching a stage Maturity and aging, where the rate of water flow is less than what was previously mentioned, which causes a decrease in the level of carving and an increase in the rates of sediment dumping, so that the river course turns into a letter "U" [1]. The question that arises here is "What kind of sediments that were thrown into the narrow corner of the letter "V" [2] (see Figure 1), and we believe that the continuous water washing process will cause the stability of some minerals eroded and fragmented by river sculpting in the upstream and downstream rocks, and these minerals are often found, including gold, and therefore it is likely that gold grains are present in more proportions. They are concentrated from anywhere, even more than the estuarine areas, in which there are many areas for gold extraction currently.

And because gold is a substance that is found in some mountains and regions on which rivers run and sweep them in its water stream, and it washes gold, fragmenting rocks and dissolving their content, then gold grains settle at the bottom of the stream before they are washed downstream, or more sediments cover them in case the river is calm and its flow rates are low Thus, the rates of sculpting are reduced.

3) Harvesting the mineral resources on the sea floor.

If the wealth in question was abundant and highly concentrated on the sea floor, and accumulated throughout history and thickened layers; Here, we need to spray or pump layers of cement resistant to sea salt and throw it on the sea

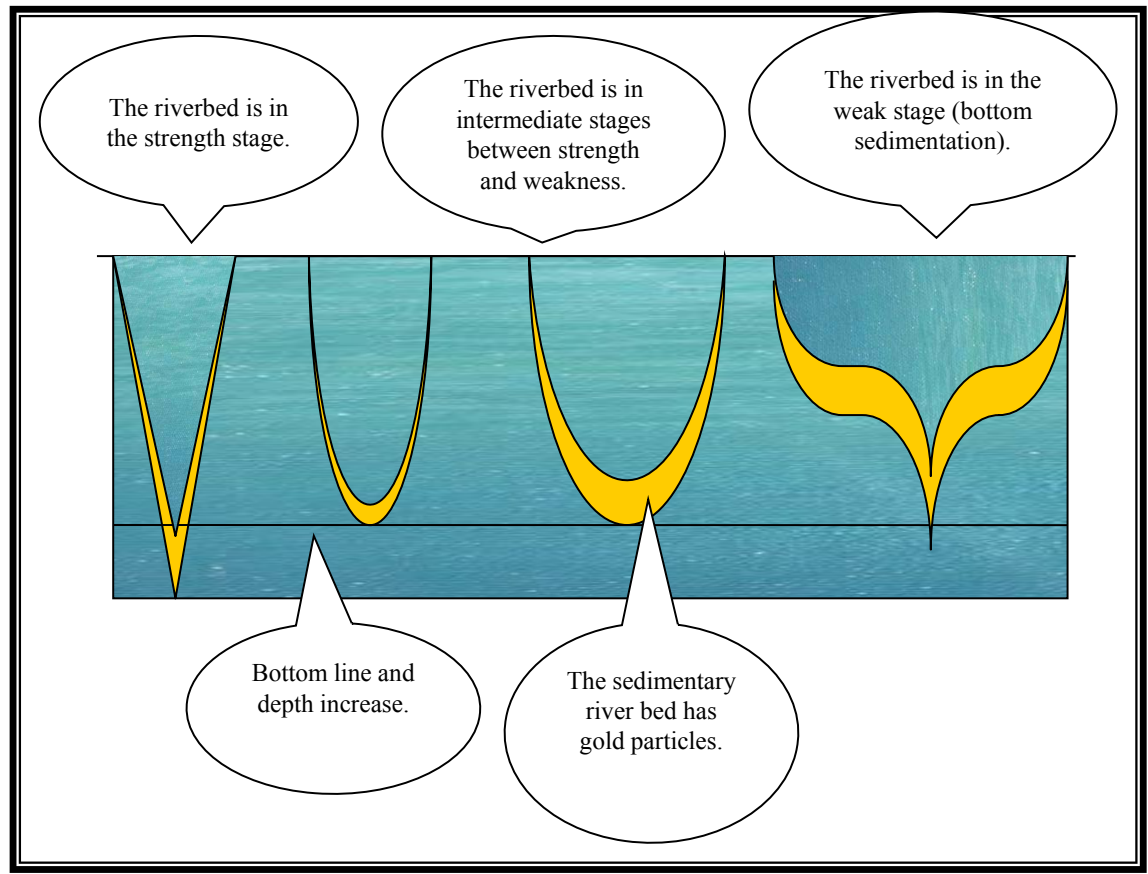

Figure 1. a cross section of the riverbed in the stages of strength (youth) and stages of weakness (aging) and the resulting change in shape, characteristics, sculpture and sedimentation, this area will be targeted by drilling and exploration operations. Source: Preparation and design of the researcher. 
floor above the target area, so that its thickness is not less than 1 meter, to begin after that drilling in the bottom, to walk under the cement layer, which will be considered here as a "protective rock shield from Deadly water pressure". It is expected that we will find many mineral resources, including petroleum and natural gas, as well as many other minerals, with huge reserves, which will increase in value when other natural resources are depleted on land.

4) Other uses will be explored by "creating safe passage" from the surface to the water floor.

In scientific research and progress: It is possible to install very different and varied scientific and military devices on the body of the building, including "temperature and salinity measurements" at different levels of the building's height submerged in water, and "wind and air pressure clock measuring devices", and contribute to knowing all Climatic changes causing or consequent on that, and "high-resolution cameras" to photograph everything that passes near the sea, whether under water or above water, which will shed more light on the migration of birds, fish and marine animals in the region, and "recording devices." For the sounds of whales and marine creatures, and "communication devices," and the building may be used to "conduct various scientific experiments", and "quarantine", and others.

It will also shed more light on the composition of sand and clay particles on the river and seabed, and monitoring the layering arrangement at the bottom of rivers and seas, and what they contain from a unique scientific record.

The importance of reaching the bottom in sports competitions: It is easy to imagine the use of the "safe access corridor in many sporting tasks and activities, including" diving "and recording world records documented in reaching the farthest distance in diving, as well as using it as a starting or ending point in" open swimming competitions at sea ", and" Fishing competitions, and others.

\section{Second: Characteristics of Drilling in Fresh and Salt Water}

This project seeks to counter the strength of water pressure that increases greatly in depths, and it is expected that many unique characteristics will appear during the operations to reach the bottom and then conduct successive drilling operations, and it will have many important effects and results, and these characteristics are:

- Characteristics of drilling in the river bed (fresh water).

It is expected that some differences will appear in drilling at the bottom of rivers that differ greatly from drilling in the bottom of the seas, oceans and lakes, due to the difference in the composition of flood sediments and the organized process of stacking their layers on top of each other, and the effects of fresh water density and its behavior in drilling and sedimentation different from salt water, and these characteristics are:

1) The grains of sand increase and their size increases as we go towards the source: The ability of the river to carry sediments and crumbs increases as 
we go towards the source, and it gradually decreases with the weakening of the water current, so it is forced to throw heavy and coarse sediments, and suffices to carry soft sediments and dissolved materials in it until it throws them downstream [3].

2) The percentage of clay, clay and fine sand (small in diameter) increases as we head towards the downstream: Because the water current weakens, as mentioned above.

3) Large rock masses are located near the sources of rivers: Because the strong river flow in its upstream causes the large rocks to wash away and push them to tens or hundreds of kilometers, until the river weakens, and throws it near the bends and folds and during its entry into the swamps and the beginnings of weak landslides, and the rest And other natural riverine obstacles.

4) An unpleasant smell of silt sediments appears during drilling in fresh water, especially whenever we head towards the downstream: Because the fine clay particles have gone through a process of decomposition and fragmentation by the action of bacteria and fungi, which causes the smell of rotting to come out.

\section{The Characteristics of Drilling in the Seabed (Salt Water)}

If we make a comparison between the characteristics of drilling in rivers and seas, we will discover many fundamental differences, unique characteristics, advantages and disadvantages in them, and in any case these differences appear in each of:

1) Finding the remains of fish structures and the present large marine mammals and extinct: Because of the weak speed and strength of the water current at the bottom, the remnants of marine organisms with a strong skeleton are not washed away.

2) Finding some minerals that have no parallel in the bottom of fresh rivers: such as manganese and others.

3) Exodus of a fish-like smell from the soil of the bottom of the seas and oceans: Because of fish residues and the rest of marine life that have accumulated over thousands and millions of years, and therefore there is a need for more ventilation.

The increased likelihood of marine soil containing iodine and some radioactive materials.

\section{Third: The Strategy for Safe Access to a Small Water Depth, Especially (10 - 20 Meters) in Rivers, through Several Balloons}

We will present here a set of alternatives and means to achieve the goal, each of which has many advantages and disadvantages, and the most appropriate ones must be chosen that are compatible with the environment and the type of actual need to reach the bottom, which are as follows: 


\subsection{Sheet Piles Strategy, the Currently Applied Engineering Method}

There is a basic engineering method in place already in place to implement "bridges and bridges bases" or any facility within a depth of up to 20 meters below water level, using "sheet piles", in their double and single sheet types (double sheet pile cofferdam - single sheet pile cofferdam). The method is based on creating a single iron fence, or creating 2 panels of fence in the water, separating them from a few meters, then pouring concrete inside, to create a strong barrier, or a circle with coherent corners, that allows conducting many activities inside it, including drilling A little bit in the water [4], but do not seek to dig tunnels at the bottom to explore for oil and so on.

\subsection{The Strategy of Using "Air Navigation Balloons" in Diving to Safely Reach the Bottom}

This is the simplest, easiest and least expensive way, so it can be done by a few ordinary people who tend to adventure and exploration, which we can call "the way of the poor to reach the bottom in shallow water", as it is fast and can be removed and reused in other places, and easy Evacuating the place of all equipment if required, and therefore it is appropriate to implement it in areas of political tension, hotbeds of war conflicts, and isolated swamp areas far from the control and management of the central government authorities.

\subsection{What Are the Specifications of the Required Balloon? How Does it Work?}

The diving balloon consists of a regular nylon balloon, of the same quality as used in the manufacture of the balloon, with some simple adjustments, and the depth used in it determines its specifications in terms of size, weight and number of layers, but several points must first be taken into account, namely:

1) Developing a preliminary vision of the environment in which the balloon is required to operate, and the goal is final, because that will determine the required characteristics, arrangements and next steps later.

2) Preparing several layers not less than 3 - 4 layers of the same size as a minimum, to reach a simple depth of no more than $10-20$ meters below the water level, with doubling the number of layers (4 - 8 layers) to reach depths between 20 and 40 meters below sea level.

3) Increase the number of layers by more than 5 - 6 layers at least as the balloon size increases in order to reach double depths in the depths of the sea.

4) Coating each balloon with several layers of rubber inside and out.

5) Inserting each balloon into the other, turning it into one heavy and multi-layer balloon.

6) Painting the outer balloon (scenes to be seen) in phosphorescent color, to clarify and announce itself to avoid collisions with ships and boats.

7) Digging a recessed belt in the heavy circle lying at the bottom of the water in preparation for the balloon's unloading. This belt will later be used to lower 
the balloon to add "several springs" to prevent water from leaking at the bottom of the balloon.

8) Measuring the diameter of the balloon's upper opening and measuring the diameter of the balloon's area, its length, width and weight after painting operations and placing the metal rings, and writing that down precisely, because that will be considered one of the important information to know the balloon's capabilities, and to understand how it works.

9) Perform the first experiment to check the ability of the balloon to prevent leakage of water.

10) Take a sample of the balloon's tissue and paint it in the same way, to explore the balloon's durability and resistance to leakage and cutting.

11) Store the balloon in a cool, not warm place, because the sun and high temperatures cause its rapid destruction.

\subsection{What Is Required for the Success of the First Realistic Bottom Balloon Experiment?}

It is better to choose a "small depth" as a start to the success of similar experiments at greater depths. Therefore, we assume that the most appropriate depth is between 10 - 20 meters, and to make sure that the bottom soil has an increase in the amount of very small particles (clay or the size of fine dust particles) and in thickness Large, and for this area to have edges close to the course, and these banks will be used to place ropes to stabilize the balloon, and of course access to the bottom must be of economic benefit, to provide some funding for the project, and to prove the actual feasibility of it.

\subsection{Actual Work Phases to Complete Successful and Effective Access beyond the Water Bed}

1) Defining and studying the target area: A study of soil, water currents, climatic fluctuations, and the nature of the general area around it. Is it possible to allocate it to any water project?, Such as reservoirs, dams, or bridges, etc.? What is the rate and type of sedimentation?, in order to determine the most appropriate and best for the success of the drilling and mining process under the water level, because the icy environment will need a heating process than other environments, and so on.

2) Throwing a large and heavy ring of concrete: It contains from the inside several belts dug inside (it will be used to place the waterproofing pins later) and it contains several lower protrusions like knives instilled in the mud, and it will prevent the balloon from moving and its drift by the water currents.

3) Putting some buffers to face the direction of the water currents: At the rate of one dam against a stable directional water stream, and of course it will increase if the water current is fluctuating in the direction (see Figure 2).

4) Lowering the balloon to the water bottom: It is expected that the balloon will float and not allow it to dive. Therefore, its bottom should be weighed as much as possible by placing some circular stones (so as not to cause a hole) in its bottom. 


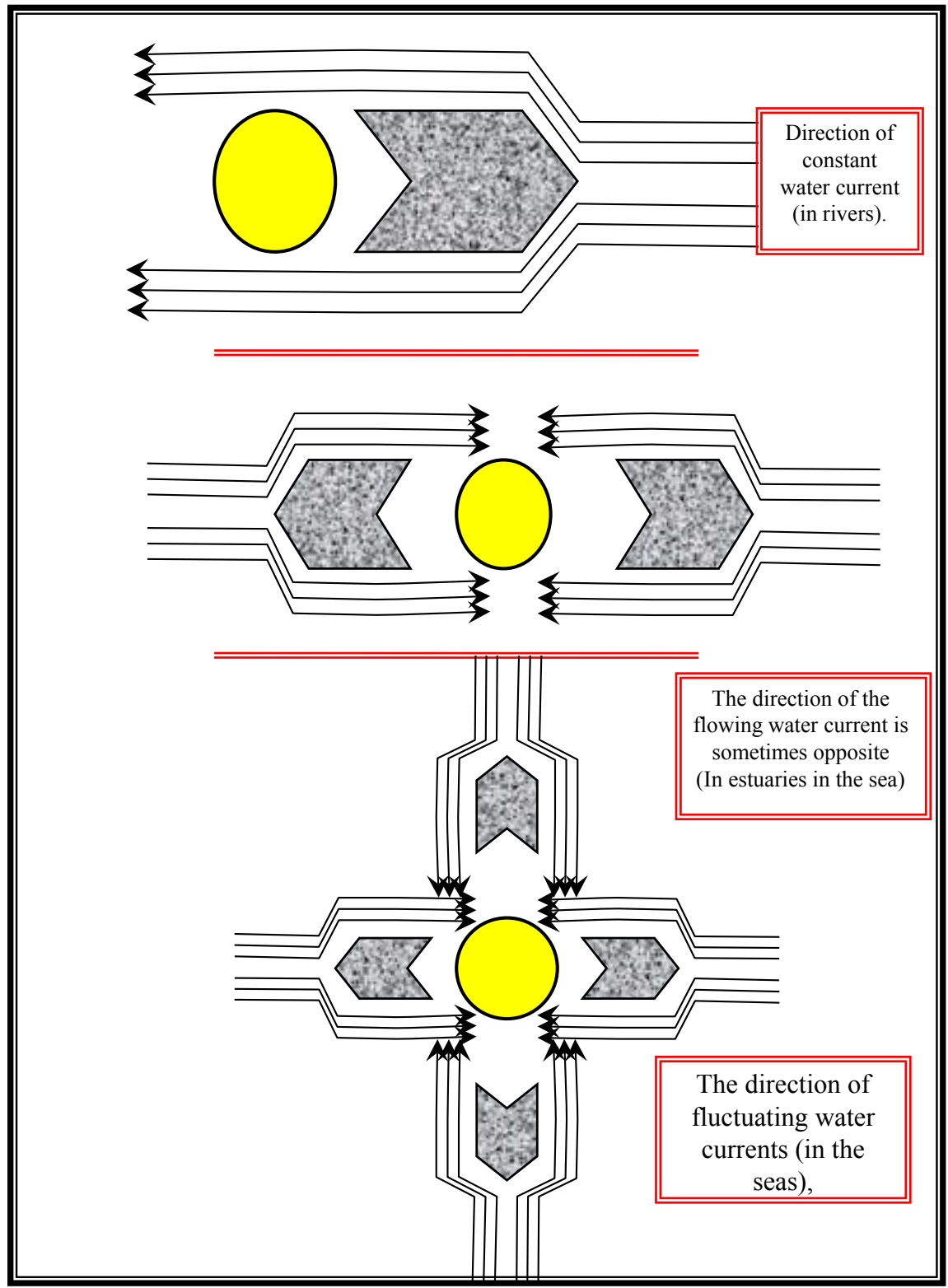

Figure 2. Characteristics of a "bumper at the bottom to resist different water currents" and how it works to protect the circuit that will be devoted to establishing the safe passage and drilling under the bottom. Source: Preparation and design of the researcher.

5) Putting rings of "metal scaffolds" in the balloon: To maintain its vertical shape without arching inward due to water pressure on the balloon, as well as keeping the largest diameter of the balloon to increase its capacity, and what will allow the entry of machines and human crews to facilitate the currency (see Figure 3).

6) Putting supports around the balloon's body to prevent its collapse or tilt and fall on the water floor: A simple engineering rule must be applied, which is: "The diameter of the struts = the same length as the balloon", that is, if the balloon reaches a depth of 10 meters, then it is required to put a pillar of 4 Directions, the length of each pillar is 5 meters, meaning the diameter of the 


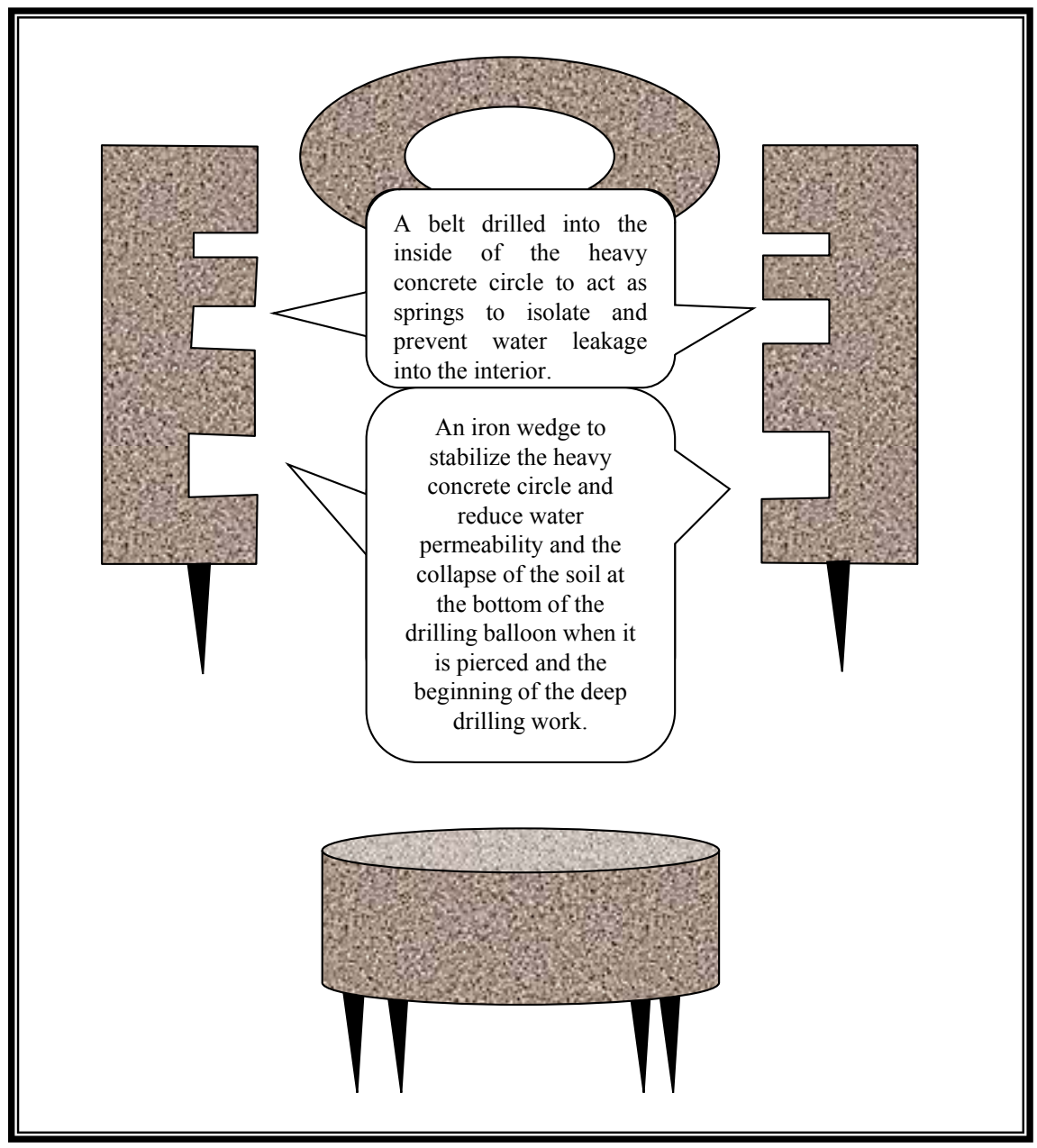

Figure 3. A cross section of a large and heavy concrete ring that will be dumped on the waterbed to receive the drilling balloon. Source: Preparation and design of the researcher.

pillars alone is 10 meters, and if the balloon reaches a depth of 20 meters, the diameter of the pillars support the balloon is 20 meters, which allows to reduce collapse in normal conditions, and of course it is possible to increase the supports in the aquatic environment The intense ocean currents.

7) Throwing fine dust particles into the balloon to form the "first layer": Because it will tighten pressure on every millimeter at the bottom of the balloon, which will prevent the flow of water from the riverbed into the middle of the drilling and working area.

8) The second layer of "sand": Because it will apply more equal pressure on each part of the clay.

9) The third layer of "circular gravel".

10) The fourth layer of "small stones" (about the size of oranges): To add more weight to make seals.

11) The fifth layer of cement: To maintain the arrangement and cohesion of the layers and not to be subjected to vinegar in distribution or arrangement (see Figure 4). 


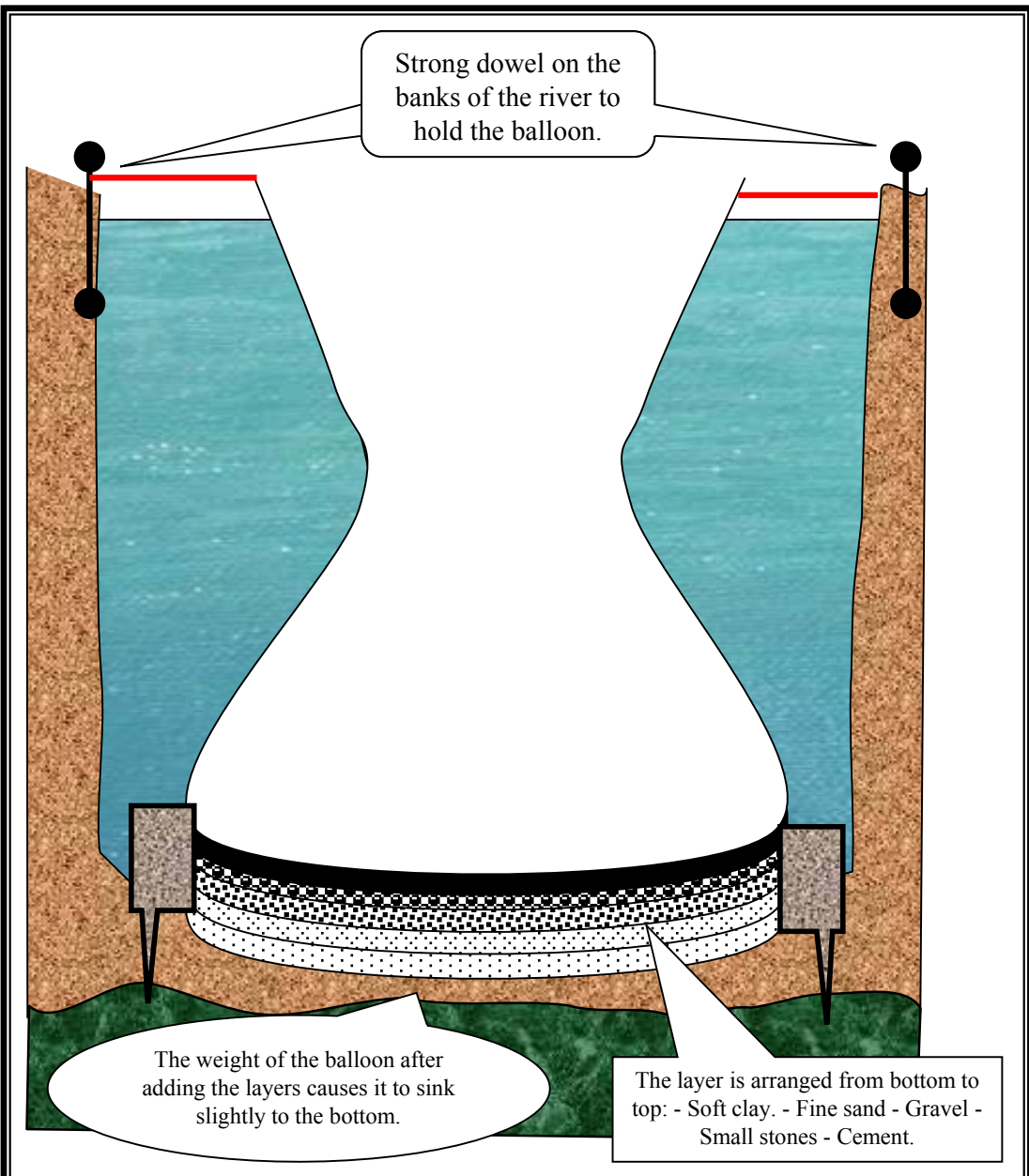

Figure 4. A cross section showing the method of fixing the balloon on fixed bearing columns and lowering it in the middle of the heavy ring with edges submerged in the mud and arranging layers of mud, sand, gravel and stones, then cement lying at the bottom of the balloon to make the process of preventing leakage when drilling. Source: Preparation and design of the researcher.

12) Drilling gently in the bottom of the balloon until its hole and reaching the bottom: It is supposed to leave 1 meter in all directions to act as a "valve to prevent water from leaking into the inside of the balloon", and drilling in the middle, and it is possible to use some wooden and iron pegs during drilling, to fix the torn parts of the balloon In the walls that will be created due to potholes.

13) Preparing border-like protective sides surrounding the drilling area: To prevent water seepage, slipping, and collapsing over the heads of workers and engineers, especially as the drilling depth increases (see Figures 5-7).

14) Preparing and installing a suction machine to collect droplets, raise them back to the surface and dispose of them: Despite all these precautions, a slight leak may occur, and therefore this is not a cause for concern, and a filter or sponge must be placed to collect only water droplets and seize the mud, because the suction of any grain of mud will follow it More of it, causing the soil to disturb below. 


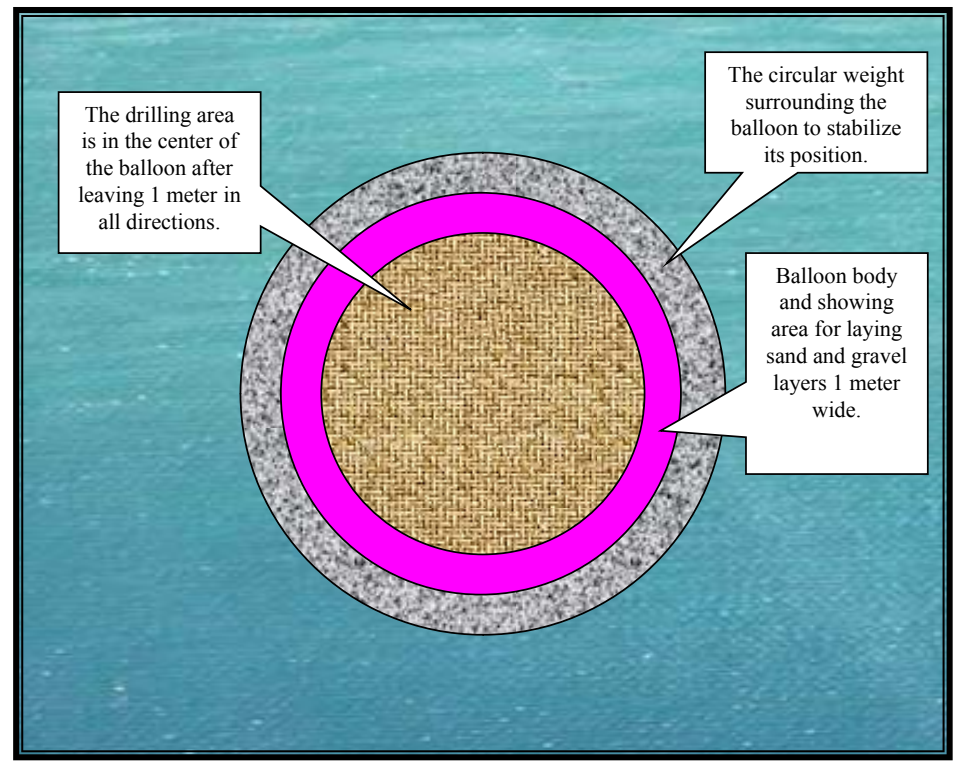

Figure 5. An upper view of the balloon after completing its position in the sea and throwing pebbles and sand, showing the drilling area in the center of the balloon. Source: Preparation and design of the researcher.

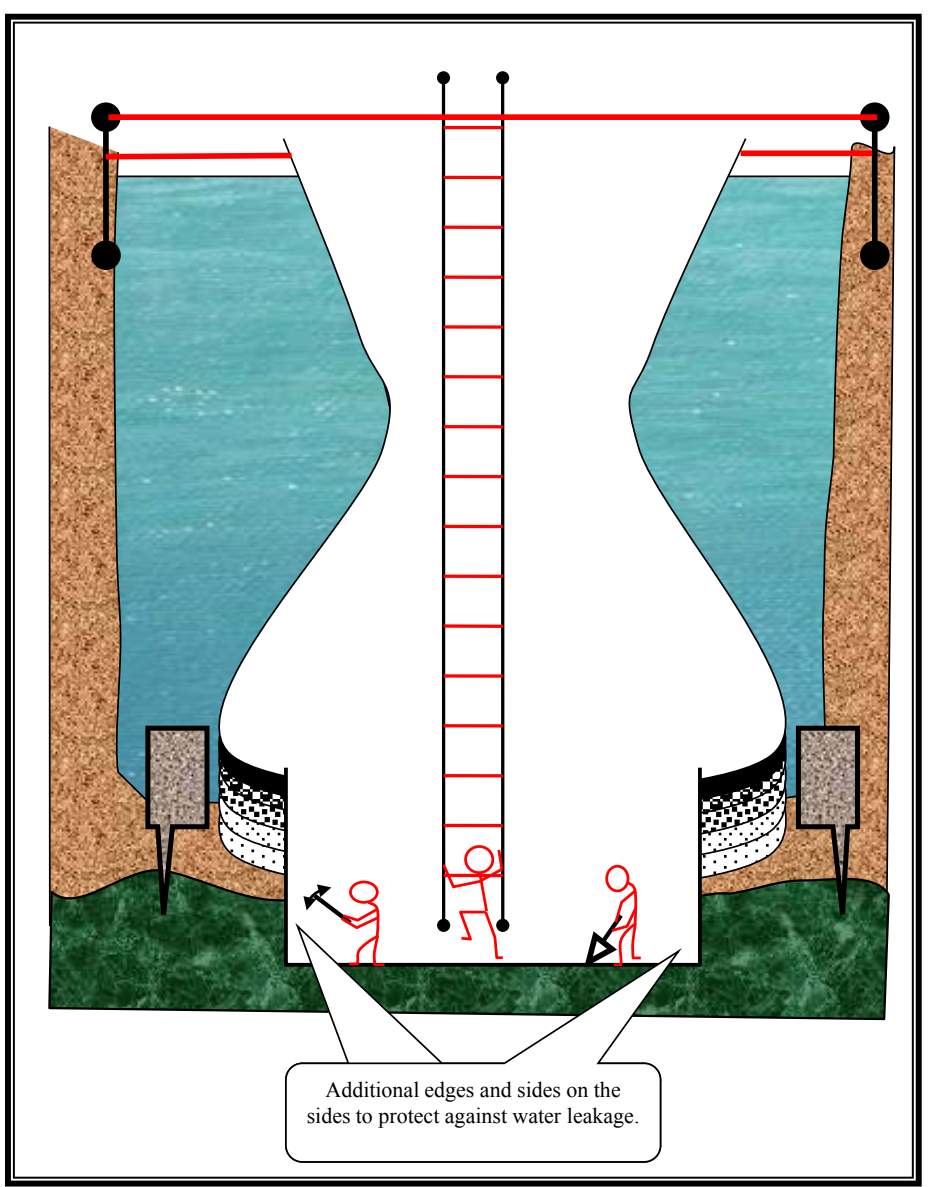

Figure 6. Drilling at the bottom of the balloon, then piercing it to reach the bottom and reach the lower layers bearing precious metal ores. Source: Preparation and design of the researcher. 


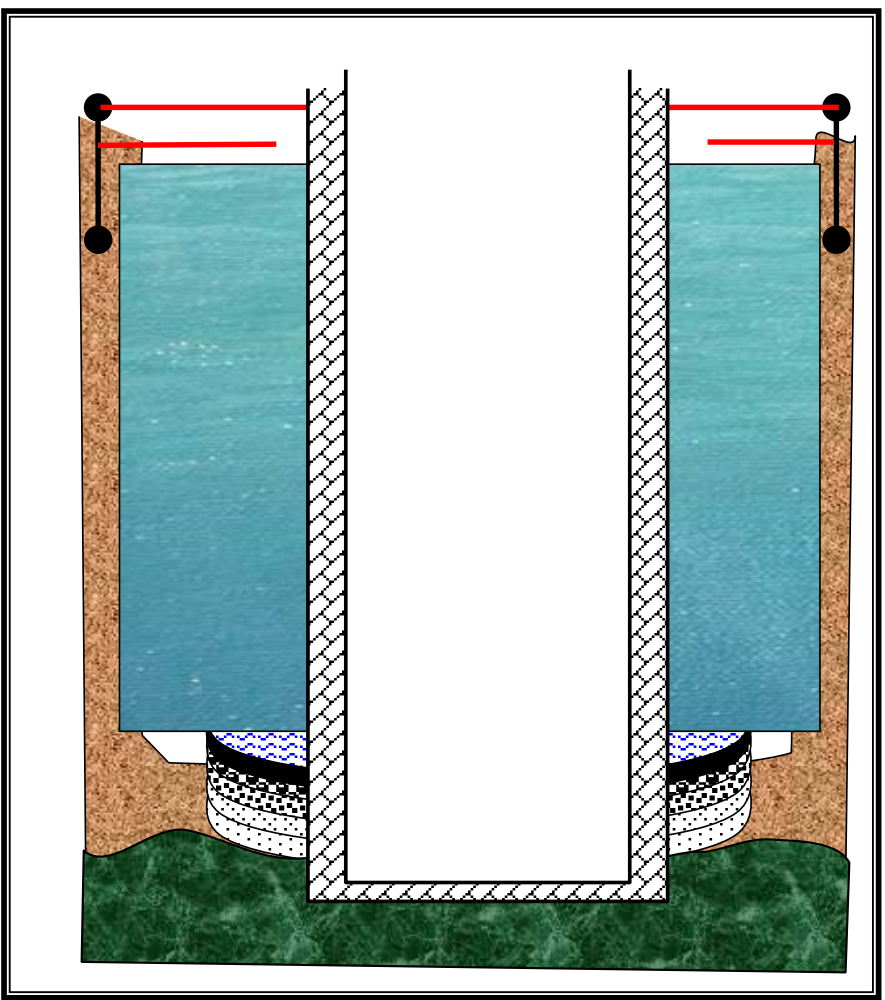

Figure 7. A cross section showing the subsequent dispensing of the balloon (except for the bottom of course) and the construction of walls to dispense with the balloon, and to achieve maximum safety and stability for the tasks of completing the excavation at the bottom. Source: Preparation and design of the researcher.

15) Preparing tubes to suck out the corrupt air and pump it outside the "balloon hole", adjacent tubes to extract fresh air from the outside and broadcast it into the depths of the cave: This method is not much different with what is applied inside regular mines, but due to the narrow space (balloon opening) it is important The direction of the "corrupt air drain pipe" should go with the wind, and the direction of the "fresh air intake pipe" facing the wind, in order to avoid mixing between the clean and the corrupt air. There is no doubt that the more work rates and the use of machines, the need for this system will increase, and it will cause a rise The noise in the balloon's opening, until it emits terrifying sounds that resemble the sound of "howling a wolf".

16) Construction of stone sides starting from the safe bottom and up to the top of the water surface: If the leakage rate decreases and the water penetration into the interior, then the need and desire to increase the safety rates to the maximum degree will be required in building a wall with cement and stones that starts from the bottom and rises to higher than the water surface level (see For Figure 7).

17) Development of the attack: That is, to continue digging, and diverting its direction to the horizontal direction after making sure of the integrity of the foundations and the soil and the strength of the tunnel's roof and its resistance to collapse (see Figure 8). 


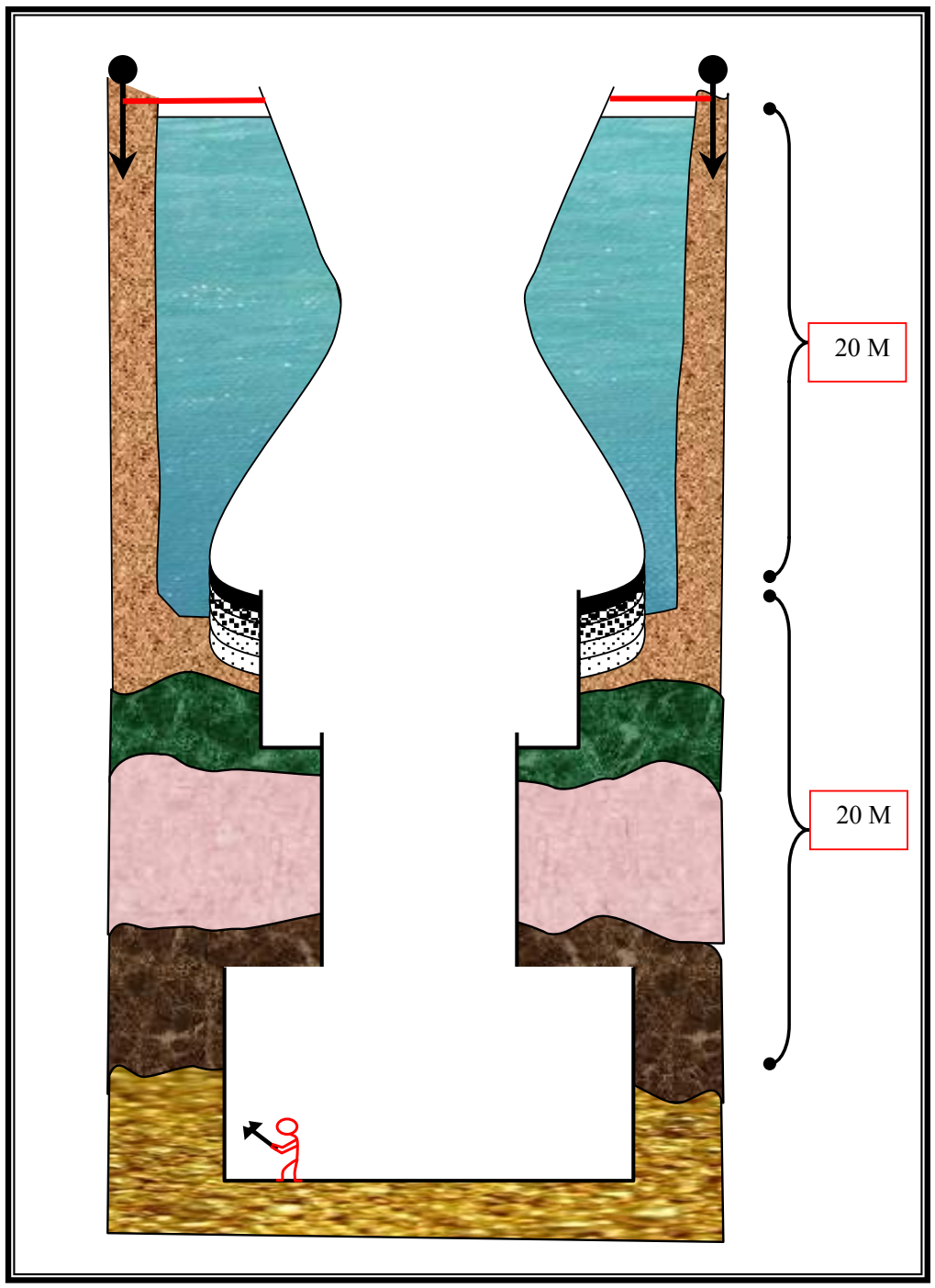

Figure 8. "Development of the attack", meaning the continuation of digging at the bottom, expanding the work area, using giant vehicles and machines, and making long paths and huge tunnels for large commercial mining. Source: Preparation and design of the researcher.

It is noted that the concrete ring contains from the inside several circular fences of regular drilling, and the upper pits will be used to put a metal filler that leads to tightening between the balloon and the circular ring to prevent the balloon movement due to the water currents, while the lower rings provided with rubber padding will prevent water leakage Into the balloon when drilling is done.

\section{Fourth: The Strategy of Drilling in Medium-Depth Water (from 20 to less than 100 Meters)}

\subsection{The Plan and Design of the Triangular Hanging Tent in What Resembles the "Indian Tents"}

A design is being planned here quite similar to the shape of the "American Indian Tents", so if we succeed in equipping 2 or 3 tents with strong fabric, and 
we can install strong supports to impede each tent inside the other, leaving a small distance of no more than 1 meter between each of them, And hanging them from above the surface of the water to hang and touch the water bottom (see Figure 9), then we removed the top of the tents to open from the top with the largest possible diameter, so that salt-resistant concrete was poured inside them, and we waited for the cement to harden; Thus, we created a "giant pear emptied from the inside", bearing the force of water pressure and some minor shocks, and it is possible to dispense with the supports that carry the tents later, but the supports will be needed in the process of transport to and from the waterbed.

And after sucking the water inside; The tent turns into a dry corridor that reaches the water floor, engineers and technicians begin to work at the bottom of the pear to pierce the bottom (see Figure 9), and "the diameter or the capacity of the opening of the upper passage" is what determines what can be involved in spending work later, even large pieces. For mechanical drilling machines it is possible to disassemble parts of them for safe entry (see Figure 10).

The bottom must be prepared to bear the weight of the "concrete pear", by making some digging if the soil is clay or sandy, but if it is completely rocky, it is possible to dispense with digging, and it is also important to pour a part of concrete with a thickness of about 1 meter, in order to resist ground subsidence, And avoid the occurrence of cracks and fractures in the pears if part of the soil falls by more than a percentage.

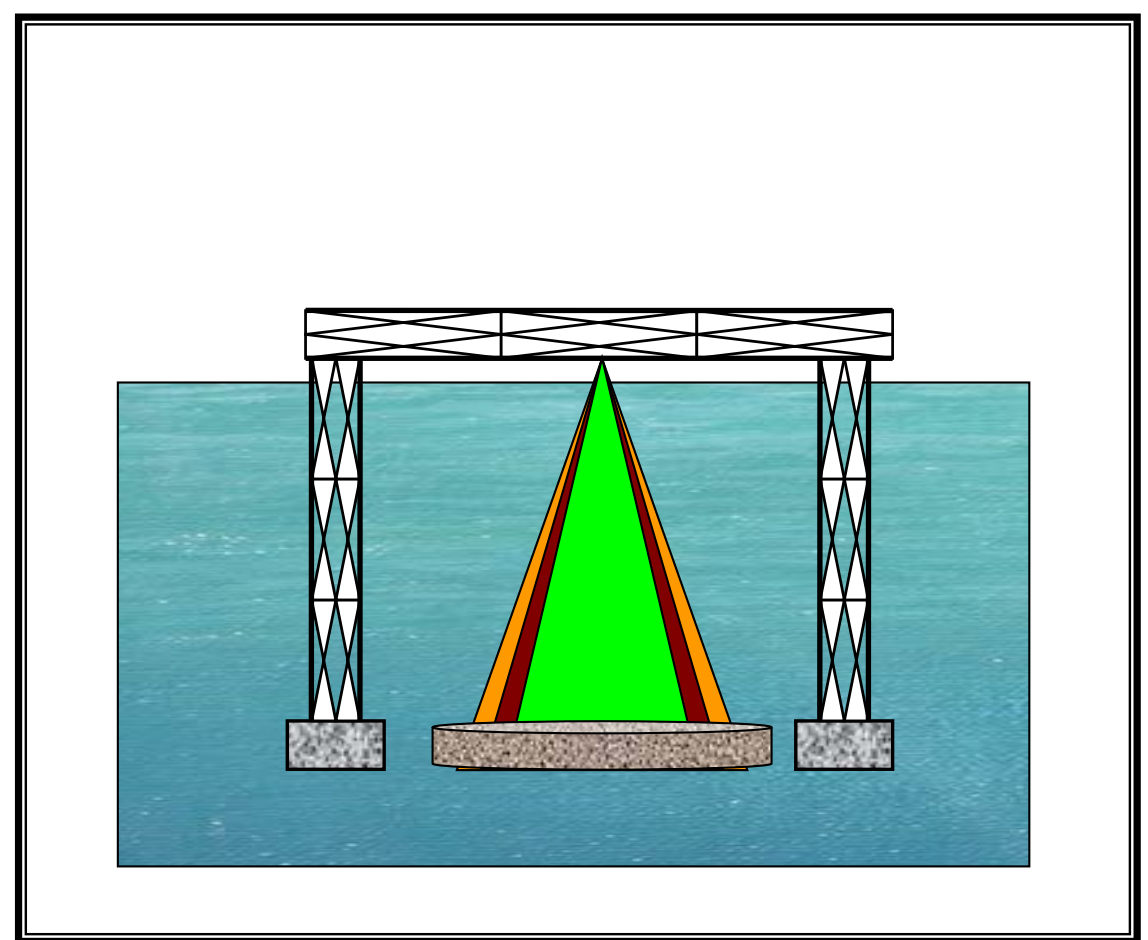

Figure 9. The three-layer open-top strategy of the American Indian Tent to pour concrete between its layers and leave its middle, so that the water is pumped out, and it turns into a safe passage to reach the bottom. Source: Preparation and design of the researcher. 


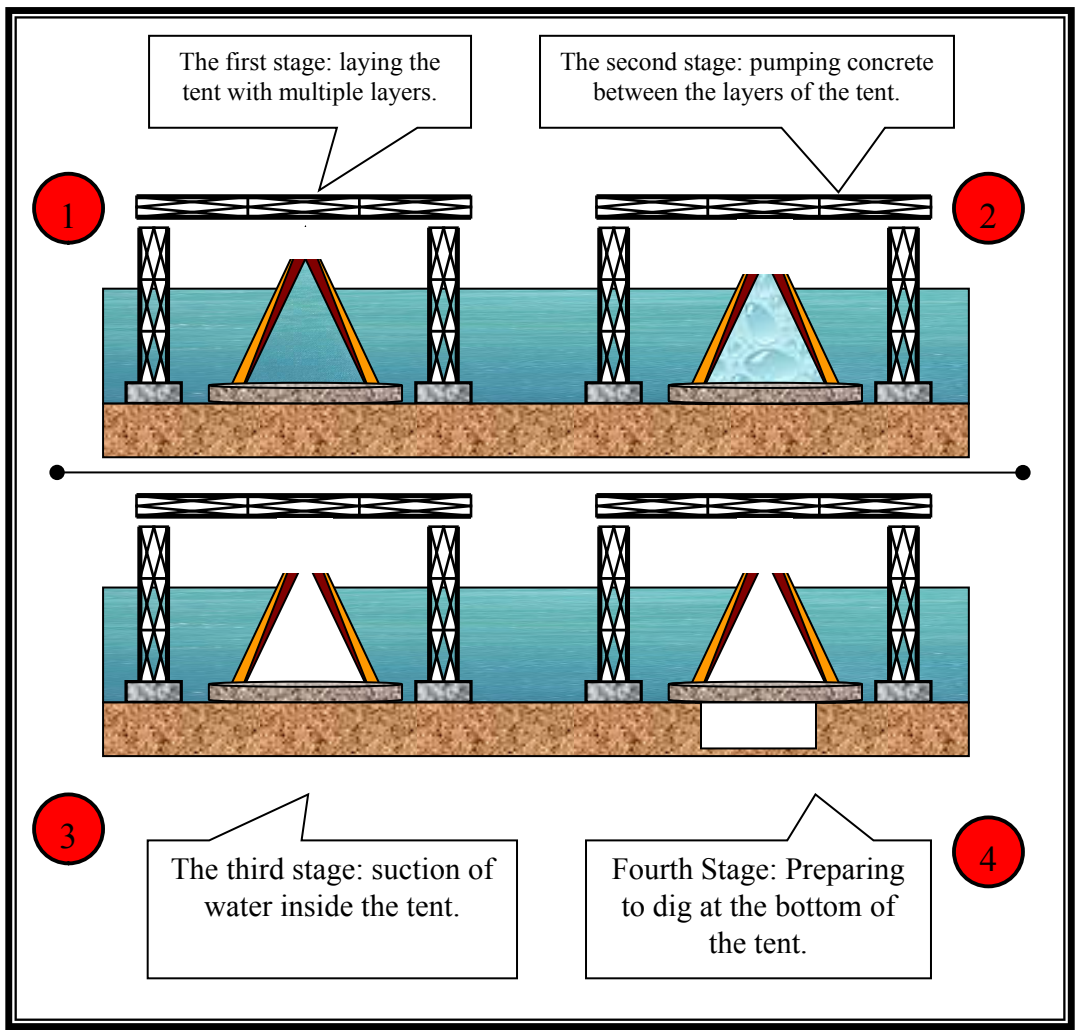

Figure 10. Stages of constructing the "Tent of the American Indians" (access corridor to the bottom). Source: Preparation and design of the researcher.

We believe that this strategy is one of the best successful tools to reach the bottom with confidence and safety, and it reduces many other problems caused by the use of balloon or concrete rings with layers of rubber, and is also suitable for fresh and salt water environments, and medium depths up to 100 meters below sea level.

\subsection{The Plan and Method of "Cascading Concrete Rings" Is Similar to a Cascading "Birthday Cake"}

We start from here using "heavy professional means" that are safer and more stable, which require the availability of great efforts that only large companies or stable countries can undertake, because they need large financial funding, organized efforts, prior planning, and elaborate and professional implementation.

It is possible to manufacture or construct a few rings of salt-resistant concrete, to be very effective in reaching the bottom, so if we assume, for example: The water depth required for safe access is 20 meters, then what is required is the creation of 3 rings, the height of one ring is 8 meters, Which means that the total is 24 meters.

\subsection{Properties of Concrete Rings}

1) The first ring lying at the bottom: Its bottom is provided with a protrusion of stainless steel pegs, and the height of the ring is 5 meters, while the width is 14 
meters, where 10 meters are left as a space that will be used as a safe passage, while the width of the concrete itself is 2 meters on the right side And 2 meters from the north side, and its upper part is equipped with circular holes surrounding the entire ring, to put a rubber belt to conduct the complete isolation process from leakage, and the ring also contains several rust-resistant metal appendages, some of which are used to raise the ring, move it and lower it by large cranes for the designated spot, and rings Sides intended for attaching buoys, and other ligaments to provide for use in tying the next ring. This ring can be dispensed with putting rubber at the bottom in contact with the bottom, if the soil is clay and sticky, where it is expected that the entire building will submerge for tens of centimeters, and maybe about $1 \mathrm{~A}$ meter or a little more (see Figure 11).

2) The middle ring: It has a total width of 12 meters ( 10 meters for the passageway, 1 meter to the right and 1 meter to the left as a shield to protect from water) and of course it is 8 meters high, and the lower and upper parts contain recessed pits surrounding each ring to isolate it when placed On the lower ring, as well as when placing the upper ring, the ring must contain the same appendages for the levers and ligatures.

3) The upper ring: 11 meters wide (10 meters dedicated to the passage, and half a meter to the right and half a meter to the left as a shield to protect from

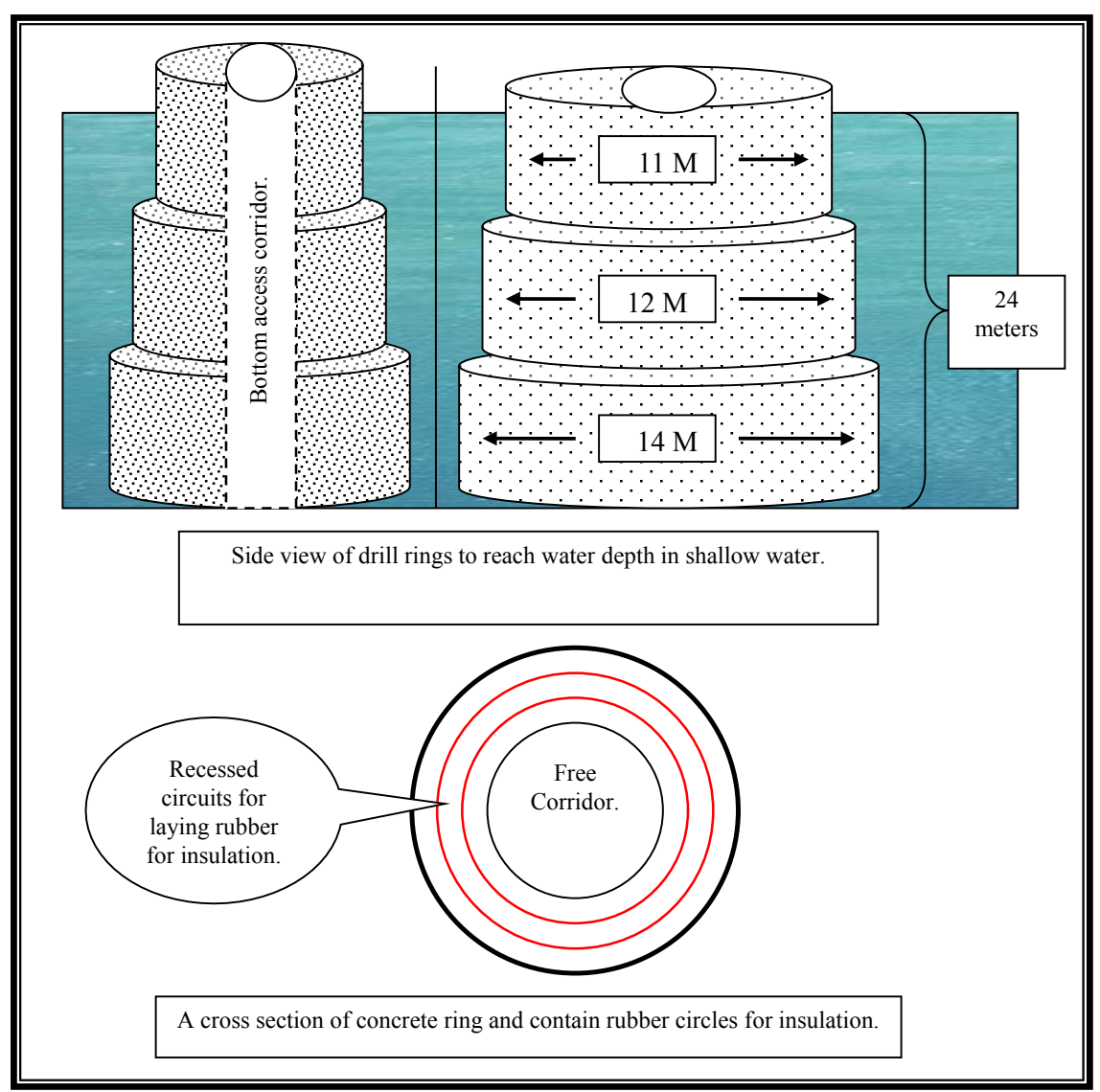

Figure 11. How to put the three rings for reaching and drilling in shallow water $(20 \mathrm{me}-$ ters). Source: Preparation and design of the researcher. 
water), and its height here must be estimated compared to the river transport in the area, to avoid the building becoming an obstacle that makes the passage difficult to the bottom, In any case, the calculations must be re-calculated after the previous two rings have been lowered and installed, knowing their ability to dive and their stability at the bottom, and the possibility of re-diving again after placing the last ring, and of course the lower part of the ring must be allocated to put the rubber to complete the isolation process, while side gaps are allocated that can be used in Installing bottom air pump pipes, electricity transmission, and others.

\section{Fifth: The Strategy of Drilling in Medium-Depth Waters (More than 100 Meters)}

It is better here to ask several questions not with the intention of answering them, but with the aim of arousing the scientific imagination and putting forward a lot of ideas, first of all, what is the number of minerals and types of rocks that have been discovered so far?, In a new opening of horizons that we do not know the limits of imagination, including the actual thinking about the permanent residence of humans under the bottom, the construction of hospitals and health resorts, and the creation of many machines and technological tools associated with the work on the project.

\subsection{The Plan for Pre-Construction of a Concrete Tower, Its Transportation and Floating in the Sea}

We believe that this strategy and the methods used and innovative are safer than lowering a tube inside a tube to drill in the waterbed, especially the seas, which is specialized only in the extraction of oil and natural gas, because this strategy reduces the level of risk of leakage that occurred, for example, in the Gulf of Mexico in 2010 [5], as well as allowing the use of all the current machines, according to what the diameter of the corridors allow to the bottom of the bottom, and will also contribute to exploring the secrets of the depths of the seas and oceans, and the extraction of minerals buried under sediments in the seabed and rivers.

This is a new challenge for engineers specializing in construction. They must choose a "shallow area", or allocate a "dry dock" to build a "hollow skyscraper from the inside", but it is not a requirement that you stop at this stage, but it is possible to build it while you are sleeping!, as if you are Adopting a long, strong-walled tunnel, with the preparation and installation of several "stainless steel rings" from the outside and inside around the building, which will later be used to suspend the various buoys to allow the entire building to float at a constant rate that does not disturb or threaten to break this huge block and split it into several parts.

\subsection{A Building Equipped with All the Construction Requirements for Human Life}

The building must contain a lot of iron to resist fracture, and also it is better to 
prepare internal corridors to lay pipes to transport water, air, electricity and sewage, and this will raise the cost of the building, and require a lot of effort, work and preparations for the occurrence of risks, and therefore the value of the building in financial terms, it must Not to abandon it, and this is what makes the process of moving it necessary and not to dispense with it or abandon it and simply blow it up if the "drilling field" is dispensed with.

\subsection{What Is the Shape of the General Building? Is it Necessary for the Building to Look Like "Ventilation Towers in Nuclear Reactors"?}

Of course not, it is possible that the hollow of the building from the inside resembles the shape of a square or rectangle or otherwise, but it is better for the exterior to be circular as possible, but why?, To facilitate the traction process later, reduce water resistance during navigation, and to slide water currents around it easily.

\subsection{The Giant Tube with a Large Base That Varied in the Characteristics of Transmission, Lifting and Pumping}

The more the base of the tube thickens and widens in a way greater than the diameter of the tube itself, the greater its ability to balance and stability, the rate of its subsidence and diving into the bottom soil (which is at the top of turbulence), and the easier it is to create passages under the waterbed soil (see Figure 12, Figure 13), when we want Allocating some places to the job of transporting each of: People - Equipment - Mining materials, and others, we will face many problems. During the construction phase: By raising and lowering the various things from the bottom to the surface, the "pear" shape will be sacrificed here, in order to create a less streamlined vertical shape, the transport stage, and the building is preserved without being broken during the stage of its lowering into the water and floating without the occurrence of faults or cracks, the installation stage in The bottom: Because its weight increases when it is installed in the water floor, and it will also cause a high rate of water leakage into the interior due to the increase in its internal area, and the difference of weight placed on parts of the giant pipe more than other parts will cause its descent from the other part, Some cracks have occurred in the building, and these are major challenges that threaten to increase the risks to the safety of the facility's work.

\subsection{Coloring and Numbering of Building Sectors as a Kind of Altimeter}

It is better to "paint the facility in a striking and bright color every few meters", as a way to facilitate the determination of the height of the building from the bottom, and to increase accuracy, it is also important to draw at least 4 belts (on the north-east-south-west side) that continue along the building to the bottom And writing down a "number next to a letter", and these simple things will provide a lot of help in the stages of building the building, launching it into the water, 


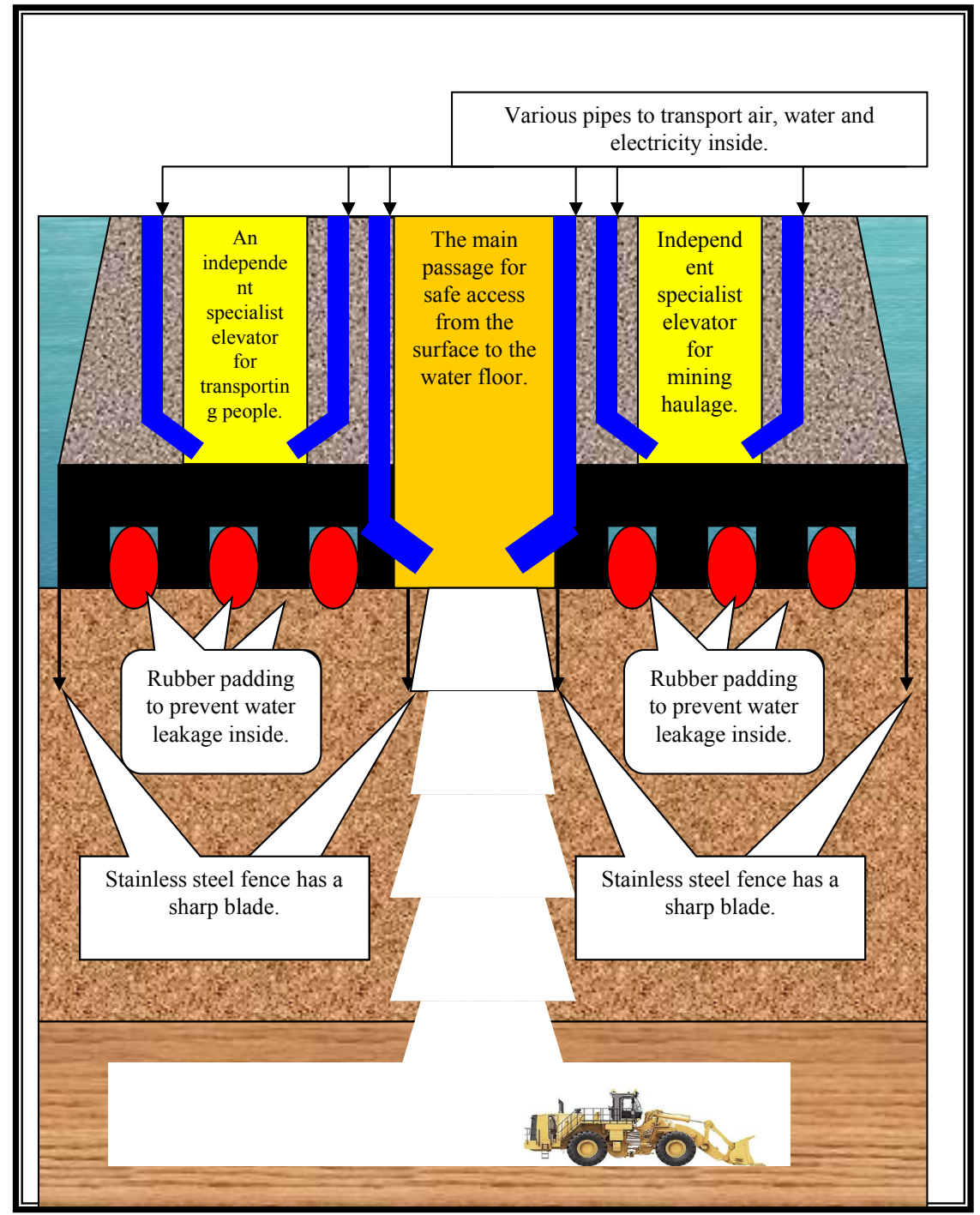

Figure 12. A cross-section of the base of the safe access tube to the water bottom (100 meters deep) and contains several gaps to add different fillings of varying strength rubber to prevent water leakage into the interior, as well as the strong iron fence surrounding the building from the inside and outside and its role in fixing it and reducing the leakage rates inside. Source: Preparation and design of the researcher.

floating it, dragging it into the sea and fixing it on the water floor, as well as it will help a lot in the relief work, maintenance, examination, etc.

\subsection{How Is the Giant Corridor Transferred to the Required Drilling Site?}

If the construction is completed, the floatation process started and proved successful, and the building stabilizes and part of it appears above the water level; So begins here the stage of shipping the building to the designated party, and it is necessary to first "install the front of the building" to resist water friction during navigation, and block the other opening (the base of the building), as well as tie several ropes in the building to prepare for tension, and perhaps it is possible to 


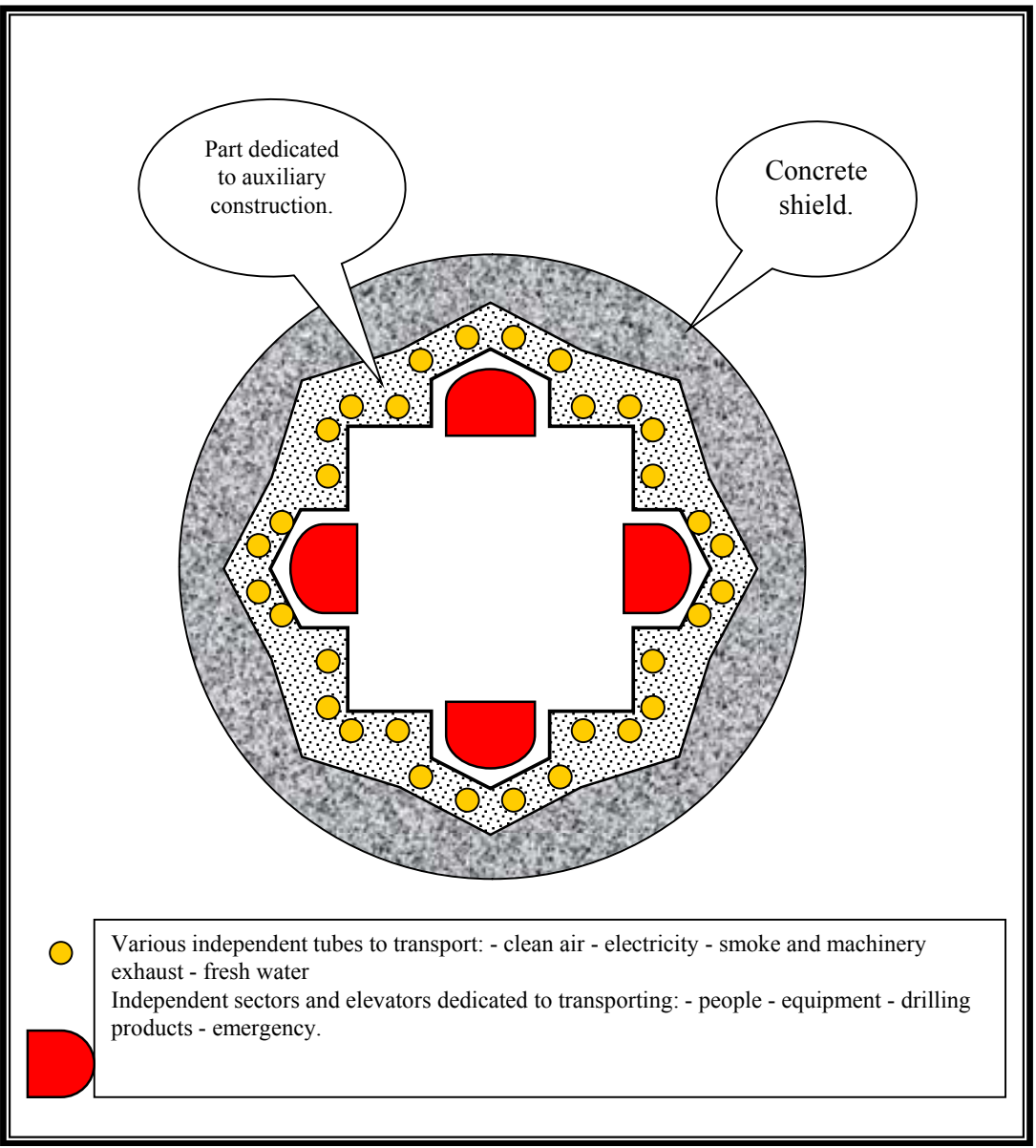

Figure 13. A cross section of the base of the diving tube for safe access to the waterbed at a depth of 100 meters or more (similar to medieval castles or blooming flowers). Source: Preparation and design of the researcher.

provide a ship or two vessels for towing, one on the right. And the other on the left, to facilitate and accelerate the traction process, it is possible to initiate a change in the method of buoyancy, so that the giant corridor takes the shape of a stable building, and then it is then dragged to the desired side

Perhaps crazy engineers would think of installing some water-propulsion equipment (Motor) in the body of the building that resembles a "giant space rocket" (see Figure 13, Figure 14) to convert it into an autonomous ship, but the problem is the possibility of the building being "broken in half" by movement Sea waves and the height of parts of the building during buoyancy and suspension of a large part in the air due to the enormity of the building compared to the width of the waves.

\subsection{All Is Well If the Project's Threatening Disaster Rate Stabilizes! How?}

What is the "general rate of water leakage and entry into the building"? What is the daily rate? Is it constant? These are the most important questions directed to building management. If this rate is stabilized, then this is complete safety. But if 


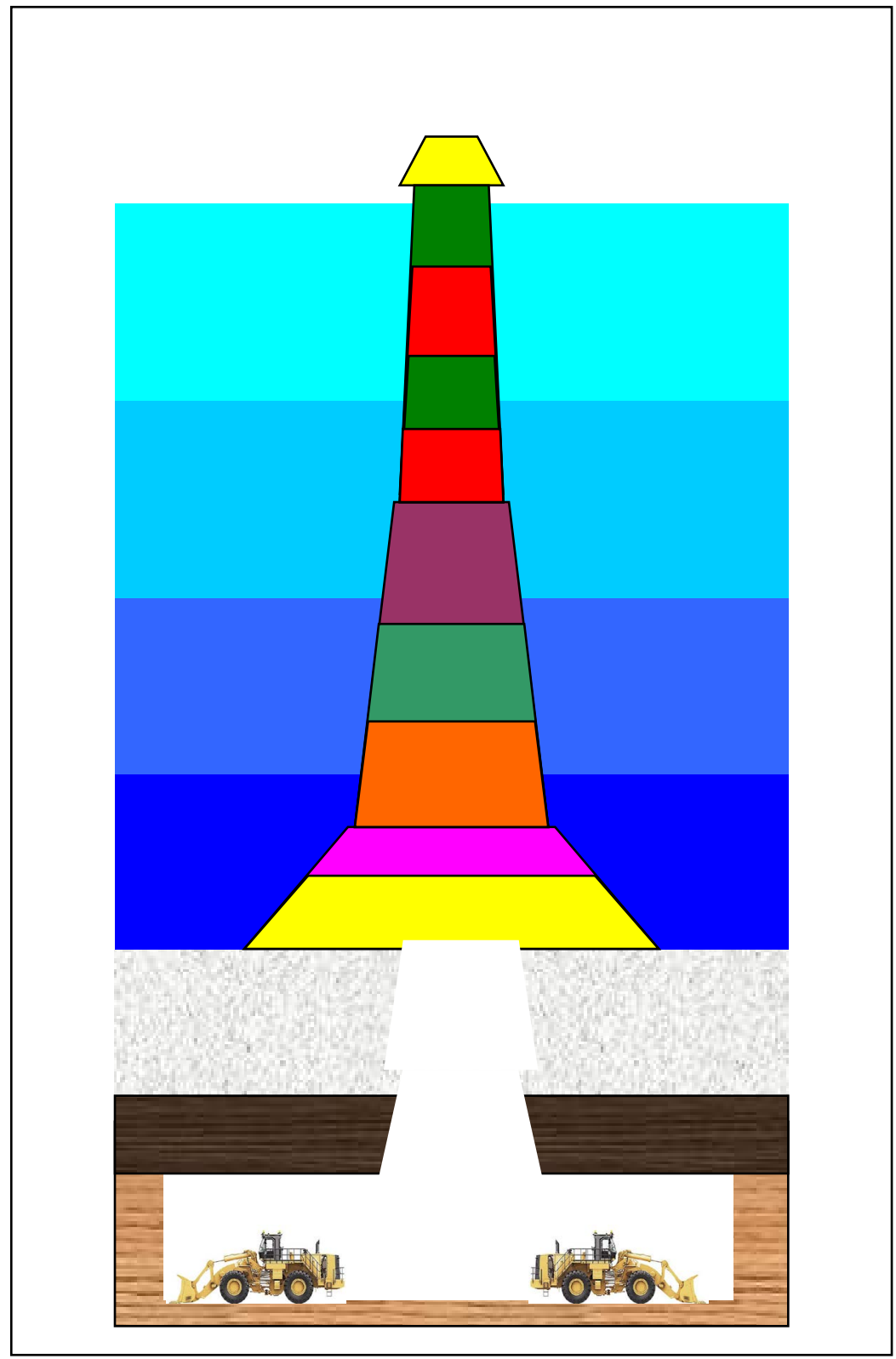

Figure 14. Cross-section of the free access corridor to the sea floor, at a depth of more than 100 meters. Source: Preparation and design of the researcher.

it increases gradually, then this means the occurrence A problem, and if it increases suddenly, then there is a disaster that may have occurred as a result of a collision with a ship or something big in the building, or a worker making a strong internal bang.

\subsection{Place 2 Stainless Iron Fences on the Bottom (Base) of the Building}

What is the shape of the required iron fence? What are its specifications?, It consists of a metal plate surrounding the building from the outside, and the second surrounds the building from the inside, and the fence must be of a strong thick- 
ness of not less than $10-20 \mathrm{~cm}$ of steel, and the blade of the fence is toothed, and the length of the fence reaches about 2 meters, and each fence must be installed. In the building with complete tightness, this strategy will greatly benefit in many respects, the first: Facilitating closing the base of the tube, as well as welding the top of the tube, by means of strong and temporary metal welding to maintain the continuation of the tube core in a dry state that water does not enter, to float and drag it into the sea The second: The iron fence will turn into the root of the building, and it will hinder its reversal, because it was planted from top to bottom thanks to the gradual disposal of buoys, and therefore it will not move except in a reverse way (by resurfacing it up). Third: The iron fence will contribute to forming an obstacle to the strong water pressure in the depths driving the water leakage into the facility, and it facilitates the drilling process at the bottom with the least amount of leakage.

\subsection{Companies Specializing in Establishing and Equipping Each Stage}

There will be a need to establish several "specialized companies" in constructing a "safe access corridor from the surface to the bottom", comprising engineers specializing in concrete and steel construction, construction, electricity and others, and a "company specializing in its transportation and installation." It includes sailors, engineers, technicians, trawlers, mobile marine cranes, wind and water suction machines. The "Building Management Company" includes specialists in management, as well as engineers, technicians and inspection and monitoring machines, and "companies to insure the building, people and equipment", and a bank specialized in managing expenses, losses and profits And "advertising and media companies" to promote the project and convey its most accurate positive news, and may conceal negative news, and a "marketing and advertising company", and others.

\subsection{A Practical Step-by-Step Model: If it Is Supposed to Reach a Depth of 100 Meters Below Sea Level, What Is Required?}

It is necessary to first measure the depth to be reached, to know the type of soil at the bottom, the degree of salinity of the water, the temperature, and the greatest height of the waves (officially registered or frequency mentioned and not scientifically proven) in this area, and give a glimpse of the surrounding environment and the risks of some cases, so are there mountains A frozen sea could cause a collision in the building? Or would huge fish like sharks and whales pass near the area? Is the region located within the most crowded tourist areas? Or, it falls within "hotbeds of war", it is likely that it will turn into a military target that is affected by acts of sabotage. Of course, the design of the building must be affected to withstand many other risks outside of the greater focus that was satisfied here with just protection from severe water pressure.

1) The height of the building: In any case, as mentioned, if the depth is 100 meters, the height of the building should increase by a few meters (higher than 
the highest level of the waves' height), it is possible that the height of the building is about 110 meters (100 under water +10 above the roof water).

2) The base of the building: The breadth of the "building base" should increase more than the capacity required for digging, so that the breadth of the base is proportional to the height of the building, to avoid being subjected to the inclination of the slightest reason, and to distribute the weight of the building over the largest flat, so its rate of decline decreases, and in any case it must be expanded The base reaches a width greater than 40 meters at least to give it the greatest amount of stability.

3) The diameter of the main access corridor from the inside and the outside: If we assume that what is required is a clearance of 10 meters for safe access to the bottom, then what is required is that the diameter of the crater reaches the concrete shield must increase by at least 1 meter in all directions, and it increases as we head towards the bottom.

4) The upper crater of the building: As was mentioned previously when the building was planned to be constructed so that the mouth width is 10 meters, then what is required is that there be a bearing wall with a diameter of 1 meter in all directions, and it is important to install valves for electronic shutdown if the weather deteriorates or strong waves occur.

5) Finishing the building process: It is possible to construct the building on land near the water, to put different pipes for use around the corridor, to take into account all the previously mentioned building conditions, and to carry out the insulation process with non-polluting oily materials, then perform a "welding process to close the passage opening from Towards the base and towards the crater", then the building was gradually pushed to the sea in deep waters, to sink to the bottom, to start after that

6) The flotation process: The building contains many metal rings that are well installed (connected to the depth of the building), which will be used to connect the floats on the various aspects of the building, and we believe that the floatation process should be carried out diagonally, to avoid splitting the building and exposing it to cracks that threaten its function and role On the assigned task.

7) The sailing process for the target area: It is done by providing a "towing ship" for the building, preferably at a time when the waves are calm and the climate is stable in the summer.

8) Putting a huge ring of concrete at the bottom surrounding the building: This ring should be almost huge in what resembles the "ring around the finger", and it is responsible for not exposing the building to inclination, and provides the greatest amount of stability in the soil and an unstable environment.

9) The installation process on the sea floor: It comes after taking a close look at the building, making sure that it is safe and that there is no rupture or crack after floating, then choosing a "stable sandy bottom area", not rocky (be- 
cause the rocky soil will not be completely flat, as It does not help in the tasks of isolation), then "freeing the building from the buoys in a gradual manner", to descend the building in a straightforward manner at the bottom, to ensure the stability of the building, to make sure that the "iron blades" surrounding the building sink to the bottom, and to re-ensure the stability of the building, and perhaps A very accurate floatation process is made to raise one side of the building and then re-lower it to stabilize it in a more balanced and safe manner.

10) Suctioning water only while avoiding the suction of a grain of sand from the bottom of the corridor: It is done by using pipes installed in the corridor, and it must be done gradually that does not raise the soil of the bottom and threaten the stability and balance of the building and threaten its fall, and a precise process must be performed to calculate the suction speed and the amount of water In the corridor and the rate of suction, to realize the presence of leakage, its size and rate, and the process continues until the bottom soil appears.

11) The first human descent of engineers and specialized technicians: For the first time here, humans (engineers and technicians) go down to measure the rate of leakage, and put a plastic ring at the bottom to be a sump to collect leakage water for suction, because the bottom soil is not suctioned and the balance of the building is disturbed.

What are the best drilling tools here?

Among the best drilling tools here are circular drilling machines, which are among the latest technologies in the world, and are suitable for most types of rocks, as well as deep drilling machines, and rock drilling machines, and it is better to use electric drilling tools, because of the lack of smoke emission of machines and mechanical equipment, and it is also important in these "Impact sensitive environment" Avoid the use of pounding machines as much as possible, if there is room to choose between rock breakers or cutting machines; The best are cutting machines, for their contribution to reducing vibrations and shocks that threaten the safety of the "drill path" work and functions.

12) Initiate drilling procedures at the bottom of the tube: The method of drilling in the form of a "repeated church bell" will be used here. In each stage drilling is done, the walls are also strengthened first by installing a stainless steel ring, and using the same ring as a drilling tool Supporting the walls at the same time, and the ring must be wide, about 1 meter high, and each ring its upper lip will be an area to collect the infusion water to re-suction and pump it outside the building, and the amount of leakage and its rate must be known and recorded first, and the bottom of the ring is considered an area New drilling, to repeat the same process, until the seepage level is stabilized, and it tends to gradually decrease as the drilling depth increases, until it is almost no longer.

13) Continuing digging to reach safe and stable soil or the rocky bottom: The drilling may continue for tens of meters, and of course the digging products are taken out, examined, and preserved in a sequential manner in which the 
depth from which it was extracted is recorded, and geologists are consulted and their opinions and estimates are determined, and the benefits of the project The digging continues until a safe soil or rocky environment is reached, then it is possible to liberate the digging process, and to dispense with the use of "church bell rings", and for free digging begins with all the usual methods, tools and machines.

14) Celebrating the success of the project and the free descent of people: The media promotion of the project and the extent of its success, and the approximate potential feasibility, should begin, so that a new phase of human work begins under the bottom.

15) Continuous management, inspection and monitoring of the building by means of remote sensing: Like any building, it will need management, monitoring and follow-up, and re-knowing the safety rates every period, and this is an important and semi-routine nature, and it is also important to use geographic information systems, especially satellites and others, to monitor the "drill corridor" after its installation, to monitor any change in the surrounding environment that threatens its safety, activity and job role.

\subsection{Underwater Drilling Strategy with Multiple Holes in What Looks Like "Wild Beaver Caves and Trenches"}

The need will arise if the tunnels become elongated and enlarged under the surface of the water, and the movement in safe passages increases, and shipping operations to and from the mine, so there is a need to create another safe passage or more of a specialized nature. The materials extracted from the mine, another for expelling smoke and exhaust of mechanical machines and all the corrupt air and extracting fresh air, another for electrical wires, control and communication devices, another for emergency and evacuation, and so on.

\section{Sixth: The Strategy of Drilling in a Marine Environment to Reach a Depth of $1 \mathrm{~km}$ or More}

This project will come as a result of previous successes that took place in depths less than that, as a kind of human ambition that increases with the passage of time, and the construction strategy here depends on some tools and aids different from the above, including each of:

- The indirect method: Thinking about using a drilling strategy in a water environment of medium depth (about 100 meters) located near the target area (with a large water depth of more than 1 kilometer), then reaching the normal drilling methods through huge and deep tunnels similar to a well to reach To this depth.

- Creating new designs that are more massive and costly: Because the required building is larger in size, and it needs many tools to aid in its stability and stability.

- The construction of a huge tower consisting of separate parts that are 
reassembled and installed above the landing point: When they are linked, they turn into a coherent, continuous block, the joints between each sector do not cause any threat to the building or water leakage.

- The construction of several ships dedicated to the assembly and installation of each sector in the building.

- Creating supporting pillars for the building: To maintain the balance of the building and prevent it from collapsing or tilting and falling on one of its sides. These pillars resemble the French "Eiffel Tower".

- The use of pumps to expel the excess water in the pipe through the drainage from specialized side openings in the building, not to raise it up: To save time and effort and facilitate the task.

- Creating pipes with side openings in the building to get rid of the exhaust exhausts that are not economically important: To avoid wasting a lot of money, efforts and electric energy in the costly lifting process, and this is possible by allocating medium or near levels near the bottom to insert carts loaded with sand into a tube. Multiple openings and different gates between the free pipe and the watery ocean, so that the last chamber is opened for the water to enter and the sand falls, then the room is closed, the water is expelled, the air pressure equalized, and so on.

- Creating dedicated openings in the building to be used as a transportation station for submarines: Because moving from the bottom to the surface will take a lot of time and effort, and because it is possible to take advantage of submarines and use them in economic fields that are not warfare; There will be a need for independent openings in the building that contain tied tubes and connect to the defective submarines after the process of expelling water and balancing the air pressure.

- General feature: The shape of the building and its functional course are similar to the special tower with a depth of 100 meters, with the addition of some piles and ligaments preventing the inclination and fall of the building, as well as the side appendages only. As for the rest of the excavation procedures, they are carried out with the same successive steps.

\section{Seventh: Safety and Emergency Strategy}

1) Safety measures for underwater drilling tunnels.

If the drilling continues, the tunnels are prolonged, and the movement of machinery, equipment, workers and drilling materials increases through the narrow exit hole, then there will be a need to implement a number of precautionary measures and measures to avoid many accidents and disasters, and among these measures are:

2) The use of a "strategy of separate movement between rooms, such as used in submarines" (the many doors between rooms and close them tightly): As it will contain any danger in specific areas and prevent its transmission to other sectors, thus allowing the protection of many places and the rescue 
of their human crews.

3) Establishing an independent pipeline network for ventilation that allows air to flow and is closed to water leakage: It is responsible for ventilating every part of the tunnel, even if the doors are all closed tightly.

4) Assignment to each drilling sector or agency an "independent system of safety plans": (See Figure 14).

5) Establishing several alternative independent corridors in case of emergency: which will be used if some paths are damaged, it is possible that some simple water leakage (drops of water fall from the ceiling regularly and without stopping) or partial collapse of the sides or roofs without leaking water, or A crack leading to a complete collapse, and these accidents happen frequently in all the mines of the world, and because the construction of the corridor increases the financial cost of the project; it should be used in collecting the drilling product as a continuation in the mining process itself, and it may be important to think about planning the construction of all the corridors so that they are next to each other or on top of each other (see Figure 15).

6) Thinking about implementing a comprehensive plan for partial or total evacuation: Through a number of procedures, protocols and various arrangements.

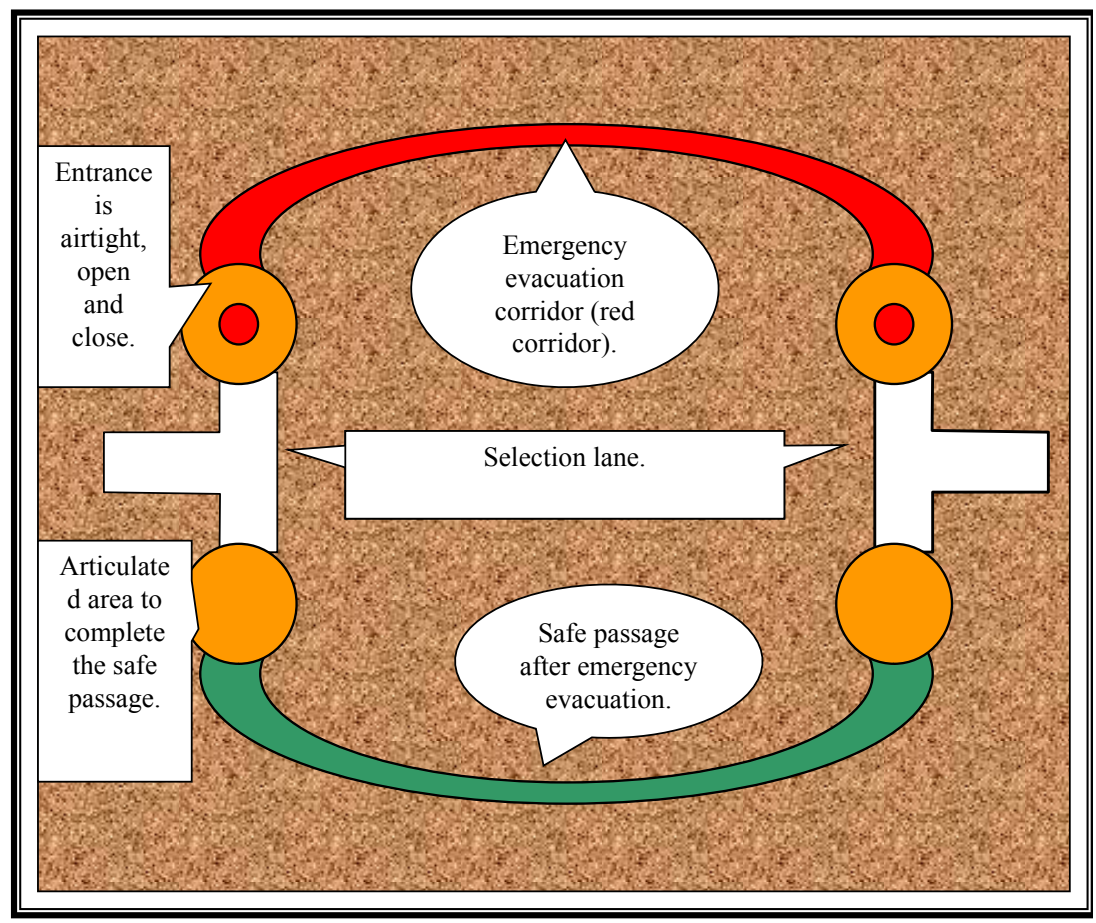

Figure 15. An upper view of the "emergency evacuation corridor" sector (the red corridor), which is a normal corridor whose mining resources have been exhausted to be transferred to retirement and use it to implement a number of evacuation procedures, and dig another corridor that tries to move away from it to the largest distance (to be safe Among the implications of the evacuation, the running out of water, and the earthquake resulting from the use of explosives in releasing the roof) to the continuation of work in the "underwater drilling mine". Source: Preparation and design of the researcher. 
7) Establishing a "Red Survival Corridor": This corridor is repeated on every side of the mine, and it is in which all the tools for survival and the lives of all workers will be located, and it contains stores for canned food, boxes filled with bottles of fresh water, some first aid drugs, and communication devices To send reports to the administrative apparatus of the mine (see Figure 16).

8) Creating several survival capsules: They are designated for the entry of individuals and transporting them to the top of the sea surface. It has a unique engineering design to escape from several deadly dangers. It must resemble a "pear", meaning it swells at the base and narrows at the top, and steel is used to create the capsule, and it is important that a shock protector be installed on its upper top as well as around the sides, and it must contain at least 3 doors, namely:

a) The first door of the capsule: On the sides (similar to the door to a room in ships), it can be closed tightly without leaking water inside, and it allows opening the door from the outside and from the inside with ease.

b) The second section of the capsule: Located at the bottom (like the well cover), which may be used if there is a need for a specialized passenger to disembark to release the capsule and complete the process of blasting the rest of the roof if the previous blasting did not meet the purpose.

c) The third section of the capsule: It is located below the second door (under the well cover), leaving a separation between them, similar to the stature of an average man $(160-170 \mathrm{~cm})$, where the diver descends and opens the stuttering door (well cover) and enters, then closes it tightly, then the door opens The third is under his feet to allow the water to enter gradually and equalize the pressure in relation to the diver's body, then he goes to the water and does his job, and this strategy makes the rest of the capsule crew safe from water.

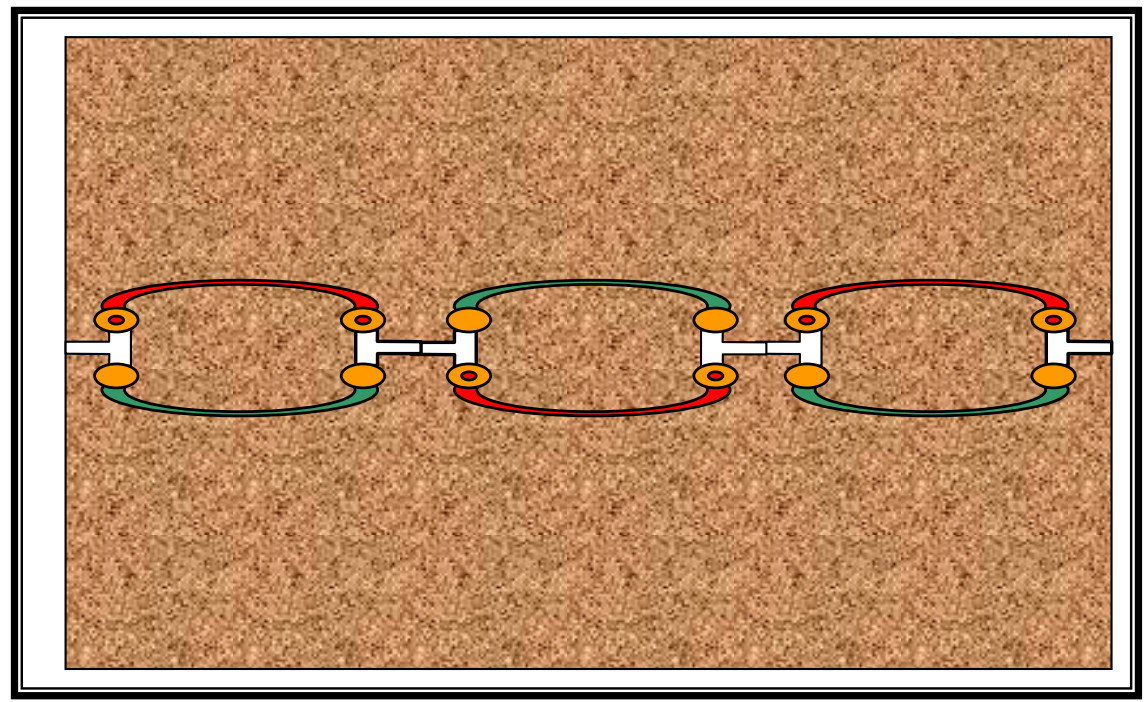

Figure 16. An upper view of the tunnels of underwater pits, their paths, their articulated parts, and their sectors assigned to retirement (next to some and not above some to avoid collapse) designated for evacuation. Source: Preparation and design of the researcher. 
These capsules contain comfortable seats firmly fixed in the capsule, belts to secure the occupants to the seat, because violent jolts are expected for the capsule before floatation, communication devices, tools and various means of survival, and it is important to put diving equipment, so one or more passengers may need to leave to complete the process. Releasing the Survival Capsule to float. Perhaps the explosives were of low effectiveness, causing a slight explosion and partially falling the roof, and the water flowed and suspended the "rescue capsule" and could not exit the narrow opening.

9) Establishing a trench with a high ceiling that is close to the top of the hole in the roof: Each sector must contain this trench, but it is better for it to be located in the first sector and the second at the end, and it is possible to add a third trench in the middle.

10) Placing "hydraulic lifting" or "effective explosives" devices in the roof of the trench: These are the final arrangements for opening a gap in the roof, by means of a "Hydraulic lift" that presses up to weaken the roof structure so that water can enter it, and it is the safest method for the tunnel system. In general, or using "explosives" to blow it up, with the possibility of damaging the entire tunnel and infecting individuals and equipment with some damage, and undoubtedly, engineers will have the ability to choose, based on the quality of the trench roof rocks, and the collapse of the trench's roof and the rocks falling in its bottom will cause water to enter, and the occurrence of Rapid water dumping, which will allow the survival capsules to move to propel upward, grab the capsule to the surface, and escape from inside.

11) Preparing compressed air drums (strong balloons) and installing them near the ceiling: By assembling a number of iron drums sealed, filled with more air, and assembling them inside iron ties in what looks like a "fishing net", and placing them on the ceiling, it may contribute to protecting The tunnel is one of the effects of destructive explosives, and the role of the air tanks will come after the opening of the smallest gap in the roof of the emergency tunnel, to ensure the air pressure in the process of strong buoyancy that causes the completion of the expansion of the roof gap, and the release of the "escape capsule". The water strongly to fill the tunnel will help activate the entire emergency system work, and if we complete the hypothetical picture of what will happen next; The set of iron drums filled with air was able to expand the opening of the tunnel's roof and rush to the roof, allowing the escape of the rescue capsule to rush, and to launch to the surface of the sea; They will be adjacent to each other, and the barrels will be considered a kind of lifeline for the capsule team when they come out to the roof.

This may take some time, perhaps more than a few hours due to the conflict between the water pressure in the depths and the group of compressed air barrels, until the remainder of the gap in the trench roof and the consequent burst of the compressed air barrel to allow the capsule to exit and float, depending on the type and installation Sand and rocks in the roof of the trench, as well as the 
thickness of the roof and the strength of water pressure.

12) Establishing a secured room with a reinforced glass facade inside the emergency evacuation trench to manage the rescue process and the evacuation stages procedures in order: A little human element is indispensable, so an engineer or a specialist must be present to manage and monitor the tasks and activities of the evacuation, even during the execution of precise blasting of the roof The trench or push it up with hydraulic lifting devices, because sometimes any equipment or tool is suspended and the evacuation process fails for the simplest reason, and therefore this room must be prepared, and an independent escape capsule attached to it, which works easily after opening the roof and the escape of the first capsules, meaning that this person will be considered a guerrilla $\mathrm{He}$ is the last to leave this section of the tunnel, without endangering his life.

13) The establishment of several consecutive independent safe passages beside the emergency evacuation corridor (red corridor) falling trench collapsed by explosives, and these corridors can be used when re-working on this side (see Figures 15-17).

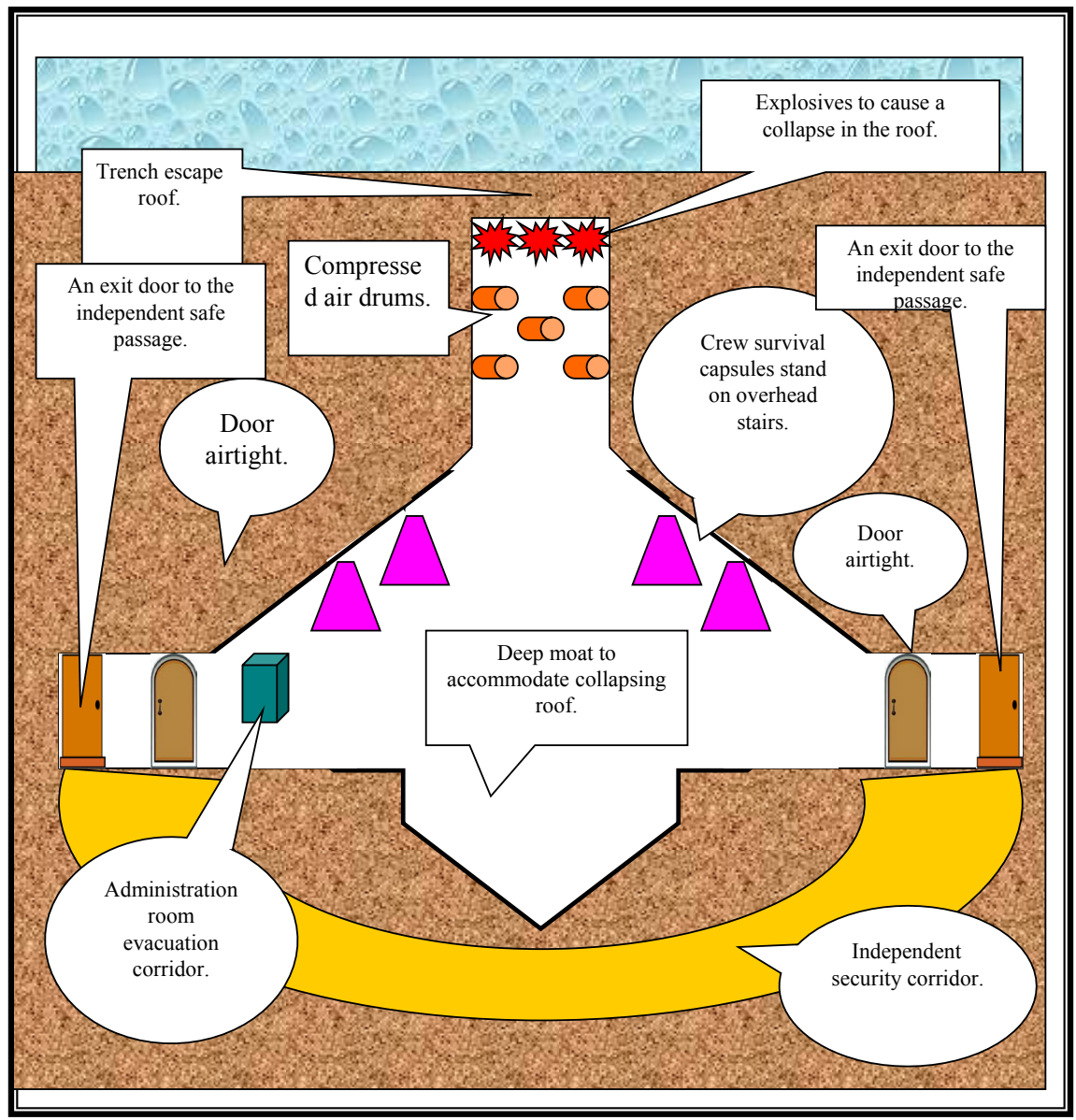

Figure 17. A side view of the evacuation corridor ("the red corridor"), which is an important part that is found on each side of the underwater drilling tunnels and their paths, which is: Part of the comprehensive plan for partial or complete evacuation. Source: Preparation and design of the researcher. 
14) Educating all workers about emergency plans, and taking precautions not to tamper with them or the occurrence of intentional acts of sabotage: This part will be left for security measures similar to the same verification or suspicion measures taken by all workers in any disciplined paramilitary zone.

\section{Eighth: Procedures for the Complete and Final Disposal of a Drilling Mine in Water}

There is a cycle to which all the various tools and projects are subject, among them the mines, each of which is an inevitable stage that calls for dispensing with its operation, and for the complete cessation of the project, it is possible to sell the well, or give it as a gift to scientific research institutions interested in exploring the depths of the earth, but it is possible to close the well by methods Pumping quantities of salt-resistant concrete [6] to close the well hole with the shape of successive church bells, and the various protrusions in these bells will prevent this cover from falling unfortunately due to the force and weight of the water weight above it, or by the force of air pressure and its attempt to exit up.

It is possible to completely dispense with every part of the group of tunnels in the well, by using explosives to destroy any apparent prominence of the underwater drilling mine, to return things to normal, and to try to return the work of nature and its various phenomena to its previous era.

\section{Effects and Potential Consequences after the Exploding of the Underwater Pit Mine}

It is expected that a large hole will erupt at the point designated for drilling under the bottom, and water will also flow in the various passages of the mine, and some effects will occur as follows:

1) In the sea: The crater will remain as it is, and the sea will continue to throw light sediments that accumulate on it over the years, such as the accumulation of air dust on home furniture, but the problem is that the penetration of sea water into the corridors will cause the possibility of activating the movement of tectonic plates and volcanic activities Seismicity if the area is volcanically active.

2) In the river: The river will dump river sediments into pits that are deeper than the normal bottom level, and this will happen quickly. It is expected that no more than one day will pass at most until the bottom is settled and the openings in the internal passages are closed (this depends on the amount of river sediments. Carried in the water current, of course), as it is expected that some large eddies will occur over the pit. The eddies may threaten river navigation slightly, but they will pose a real danger to the swimming of ordinary people.

\section{Conclusions}

It is a great opening for humans by entering a completely new environment and field that has not been previously touched upon, and this research will contribute to increasing exploration for natural resources in the depths of fresh and 
salt water, and in shallow water areas and very deep areas, and it will lead to an increase in the extraction of petroleum, natural gas, gold and others. The increased exploration process will contribute to lowering prices, and may lead to the discovery of more minerals and other resources. Rather, it will open up a new space and environment for people to perform all activities and roles similar to what is happening in the current environments.

These detailed drawings, accurate engineering designs and explanatory visions are adjustable, switched and developed, and it must be in line with different environments, and to keep pace with the changes that will occur in the future.

\section{Conflicts of Interest}

The author declares no conflicts of interest regarding the publication of this paper.

\section{References}

[1] Judeh, G.H. (1995) Physical Geography. Al Ma'arif Press, Alexandria.

[2] Salahal-Din, B. (1996) Principles of Physical Geography. Contemporary Thought House, Beirut.

[3] Guda. (1995) Physical Geography. Al Ma'arif Press, Alexandria.

[4] Ahmed, I. (2005) Concrete Bridge Design. Benha University, Egypt.

[5] Saber, M. (2015) Human and Environmental Pollution. General Administration for Scientific Recommendation and Publishing, King Abdul-Aziz City for Science, Saudi Arabia.

[6] Imam, M. (1995) Properties and Testing of Materials (Cement). Cairo University, Cairo. 